\title{
The influence of viscosity on the frozen wave instability: theory and experiment
}

\author{
EMMA TALIB, SHREYAS V. JALIKOP AND ANNE JUEL \\ Manchester Centre for Nonlinear Dynamics and School of Mathematics, \\ The University of Manchester, Manchester M13 9PL, UK, \\ anne.juel@manchester.ac.uk.
}

(Received 18 August 2006 and in revised form 26 February 2007)

We present the results of an experimental and linear stability study of the influence of viscosity on the frozen wave (FW) instability, which arises when a vessel containing stably stratified layers of immiscible liquids is oscillated horizontally. Our linear stability model consists of two superposed fluid layers of arbitrary viscosities and infinite lateral extent, subject to horizontal oscillation. The effect of the endwalls of the experimental vessel is simulated by enforcing the conservation of horizontal volume flux, so that the base flow consists of counterflowing layers.

We perform experiments with four pairs of fluids, keeping the viscosity of the lower layer $\left(v_{1}\right)$ constant, and increasing the viscosity of the upper layer $\left(v_{2}\right)$, so that $1.02 \times 10^{2} \leqslant N_{1}=v_{2} / v_{1} \leqslant 1.21 \times 10^{4}$. We find excellent quantitative agreement between theory and experiment despite the simple model geometry, for both the critical onset parameter and wavenumber of the FW. We show that the model of Lyubimov \& Cherepanov (Fluid Dyn. vol. 86, 1987, p. 849), which is valid in the limit of inviscid fluids, consistently underestimates the instability threshold for fluids of equal viscosity, but generally overestimates the threshold for fluids of unequal viscosity. We extend the experimental parameter range numerically to viscosity contrasts $1 \leqslant N_{1} \leqslant 6 \times 10^{4}$ and identify four regions of $N_{1}$ where qualitatively different dynamics occur, which are reflected in the non-monotonic dependence of the most unstable wavenumber and the critical amplitude on $N_{1}$. In particular, we find that increasing the viscosity contrast between the layers leads to destabilization over a wide range of $N_{1}, 10 \leqslant N_{1} \leqslant 8 \times 10^{3}$. The intricate dependence of the instability on viscosity contrast is due to considerable changes in the time-averaged perturbation vorticity distribution near the interface.

\section{Introduction}

Interfacial instabilities occur in many multi-layer flows present in processes of industrial significance, such as solvent extraction, coating and oil recovery, and may lead to important changes in the heat and mass-transfer properties of the system (Roberts et al. 2000). Oscillatory forcing, in particular, can have very significant and sometimes unexpected effects on fluid interfaces. It is well known, for example, that the vertical vibration of a stably stratified two-layer system leads to the parametric excitation of the interface, known as the Faraday instability (Kumar \& Tuckerman 1994). However, when the heavier layer is placed on top of the lighter fluid, so that the configuration is gravitationally unstable, vertical vibration has been shown to stabilize the resulting Rayleigh-Taylor instability (Wolf 1969, 1970). 
Under horizontal, sinusoidal oscillation, stably stratified layers of immiscible liquids are differentially accelerated due to their density contrast. If the liquids are contained in a vessel with endwalls, the shear flow generated by the periodic forcing consists of counterflowing layers, since the liquids are incompressible. This configuration was first addressed by Wolf (1969), who observed a spatially periodic waving of the interface, steady in the frame of reference of the oscillating vessel, which is often referred to as a 'frozen wave' (FW). Subsequent experimental studies have focused primarily on the onset of the FW instability compared to the analytical stability predictions of Lyubimov \& Cherepanov (1987), valid in the limit of vanishing viscosity in both layers. In their model, two superposed, horizontal layers of fluid of infinite lateral extent and equal height, $h=d_{1}=d_{2}$ (where $d_{1}$ and $d_{2}$ denote the heights of the lower and upper layers respectively), are contained between horizontal, rigid plates and subject to a horizontal vibration, $a \cos \left(\omega t^{*}\right)$, where $\omega=2 \pi f, a$ and $f$ are the amplitude and frequency of vibration respectively, and $t^{*}$ denotes time. In order to model the stability of counterflowing layers, which occur in vessels of finite length, they imposed the conservation of horizontal volume flux. They considered the limit of large frequencies and vanishing amplitudes of forcing, where fast oscillatory motion can be linearly decoupled from the mean flow, to yield, on average, a steady pressure gradient contribution to the mean flow equations. A linear stability analysis indicates that a perturbation of dimensionless wavenumber $k$ to the flat interface becomes unstable if the vibrational parameter, $W=(a \omega)^{2} /(g h)$, where $g$ is the acceleration due to gravity, exceeds

with

$$
W>\frac{1}{2} W_{\gamma}\left(\frac{k}{k_{\gamma}}+\frac{k_{\gamma}}{k}\right) \tanh k,
$$

$$
W_{\gamma}=\frac{\left(1+R_{1}\right)^{3}}{R_{1}\left(1-R_{1}\right)} k_{\gamma}^{-1},
$$

where $R_{1}=\rho_{2} / \rho_{1}$ and $\rho_{i}(i=1,2)$ is the density of the lower and upper layers respectively. For layer heights $h>\sqrt{3} l_{c}$, where $l_{c}=\sqrt{\gamma /\left(\left(\rho_{1}-\rho_{2}\right) g\right)}$ is the capillary length and $\gamma$ the interfacial tension, the instability is of finite wavelength and the critical wavenumber $k_{\gamma}=h / l_{c}$ is determined by the capillary length. The dispersion relation given by equation (1.1) is analogous to that of the classical Kelvin-Helmholtz instability, where short-and long-wavelength perturbations are suppressed by the stabilizing effects of capillary and buoyancy forces respectively. Thus, in the absence of viscosity, the capillary length is the natural length scale of the instability. Khenner, Lyubimov, Belozerova \& Roux (1999) extended the inviscid analysis of Lyubimov \& Cherepanov (1987) to arbitrary amplitudes and frequencies of forcing. Similarly to Kelly (1965), who considered layers of infinite depth, they reduced the inviscid problem to a Mathieu equation. Thus, they demonstrated the existence of a parametric instability in addition to the Kelvin-Helmholtz instability uncovered by Lyubimov \& Cherepanov (1987).

Ivanova, Kozlov \& Evesque (2001) performed experiments with a perfluorinated liquid, FC-40, and machine oil. By varying the frequency and amplitude of forcing, they found that the critical value of the vibrational parameter for the onset of frozen waves, $W$, monotonically decreased toward a constant threshold, in good agreement with the stability prediction of Lyubimov \& Cherepanov (1987), for non-dimensional frequencies $\Omega=\left(\omega d_{2}^{2}\right) / \nu_{2}>200$, where $v_{2}$ is the kinematic viscosity of the upper, more viscous layer (machine oil). Wunenburger et al. (1999) used liquid $\mathrm{CO}_{2}$ in equilibrium with its vapour near the critical point. They also varied the distance 
from the critical point, i.e. the density contrast and the interfacial tension, but were unable to perform onset measurements. Their measurements of the wavelength of the frozen wave are consistent with the results of Lyubimov \& Cherepanov (1987), although they do not reach quantitative agreement even for high frequencies of forcing. They estimate that for high frequencies, the boundary layers are sufficiently thin to be negligible compared to the wavelength of the disturbance, but that the dimensionless amplitude of forcing is not vanishingly small, since $a / d_{2} \sim k_{c}^{-1}$, where $k_{c}$ is the critical wavenumber. Independently, González-Viñas \& Salán (1994) performed similar experiments where they observed both frozen and travelling waves. They suggest that the reduction of the wavelength at onset with increasing frequency of forcing is linked to the walls of the vessel acting as wave generators. Finally, recent measurements of the dependence of the marginal value of $W$ on the wavenumber in miscible liquids by Legendre, Petitjeans \& Kurowski (2003) appear consistent with the predictions of Lyubimov \& Cherepanov (1987) with $\gamma=0$, although they do not invoke a selection mechanism for the critical wavenumber. Surprisingly, they do not detect any influence of viscosity, using water in the bottom layer and two waterglycerine mixtures of dynamic viscosity $0.07 \mathrm{~kg} \mathrm{~m}^{-1} \mathrm{~s}^{-1}$ and $1.0 \mathrm{~kg} \mathrm{~m}^{-1} \mathrm{~s}^{-1}$ respectively in the upper layer.

Thus, previous experiments have focused primarily on varying the amplitude and frequencies of forcing for a few isolated values of the viscosity ratio, $N_{1}=v_{2} / v_{1}$, where $v_{1}$ is the kinematic viscosity of the lower layer. The disparity between existing experimental results emphasizes the need for a comparative study between carefully controlled experiments and a viscous stability model, in order to establish the details of the mechanisms underlying the onset of the FW over the range of nondimensional frequencies, $\Omega$, and amplitudes $c=a / d_{2}$, accessible experimentally. In the $\mathrm{CO}_{2}$ experiments, both layers have similar kinematic viscosities, whereas in the remaining experiments the fluid layers are typically chosen to have widely differing viscosities. In the experiments of Ivanova et al. (2001), the viscosity ratio between the layers is $N_{1}=93.5$. We have found in similar fluids that relatively large values of $N_{1}$ were necessary to avoid the emulsification of the oscillating interface, noted by Ivanova et al. (2001), for values of $W$ less than those required for the onset of the frozen wave. Furthermore, motivated by Berman, Bradford \& Lundgren's (1978) study of two-fluid spin-up, Shyh \& Munson (1986) find surprisingly low critical values of the amplitude and frequency for viscosity ratios as large as $N_{1}=10^{4}$ in a related geometry consisting of an oscillating cylinder. Careful onset measurements have also recently been performed in this geometry for $N_{1}=10^{4}$ by Yoshikawa (2006). We choose to focus on uncovering the effect of viscosity on the FW instability. Specifically, we demonstrate that altering the viscosity contrast can either lower or raise the threshold for the onset of the FW depending on the value of $N_{1}$.

The effect of viscosity on the steady Kelvin-Helmholtz instability has been addressed analytically by both Lindsay (1984) and Hogan \& Ayyaswamy (1985). They find that introducing viscosity to one of the fluid layers leads to the reduction of the critical velocity difference between the layers, by a factor which depends solely on the density ratio between the fluids. A comparison between inviscid and viscous two-layer stability models of the steady shear of a liquid-air interface is presented by Barnea \& Taitel (1993). Somewhat surprisingly, it indicates that the neutral stability curves for low values of the liquid viscosity differ significantly from the inviscid result, and that the inviscid model appears in closest agreement with the predictions for viscous liquids. Finally, Hinch (1984) proposes a physical mechanism based on the increase of vorticity disturbances near the interface with viscosity contrast to explain 
why two-layer Couette flow of equal-density fluids, but different viscosities, and with no interfacial tension is always unstable to a short-wavelength instability (Hooper \& Boyd 1983).

We study the linear stability of two superposed fluid layers of arbitrary viscosity and infinite lateral extent, subject to horizontal vibration of arbitrary amplitude and frequency, and we enforce the global conservation of horizontal volume flux. Our primary aim is to assess the relevance of this simple geometry to the experimental situation through direct comparison. In contrast with the Faraday configuration studied by Kumar \& Tuckerman (1994), the horizontal vibration of the vessel generates a time-periodic basic shear flow, so that the spatial dependence of the linearized perturbation equations cannot be solved analytically. Thus, the inclusion of viscosity into the model requires the numerical solution of the perturbation equations. Khenner et al. (1999) used finite differences to approximate the spatial dependence of the flow and a Fourier expansion to resolve the time-dependence. They focused on equalviscosity layers, i.e. $N_{1}=1$, but could not resolve low-viscosity flows with nondimensional frequencies $\Omega>360$. We employ a pseudo-spectral discretization scheme to approximate the spatial dependence of the perturbed flow field. To resolve the time-dependence of the solution, we adopt an iterative procedure based on Floquet theory developed by Or (1997) in his study of the stability of a horizontal liquid layer on an oscillating plane. This enables us to resolve the flow over a wide range of non-dimensional frequencies and viscosity contrasts. In this paper, we present results with non-dimensional frequencies, i.e. the ratio of vibrational to viscous forces, in the lower layer of $1.6 \times 10^{2}<\Omega_{1}=\Omega N_{1}<2 \times 10^{5}$, and in the upper layer of $8 \times$ $10^{-1}<\Omega_{2}=\Omega<5 \times 10^{4}$. The viscosity ratios studied are in the range $1.0<N_{1}<$ $6.0 \times 10^{4}$. The ratio of vibrational to capillary forces is consistently large with $4.0 \times 10^{3}<W e<1.0 \times 10^{5}$. In this limit, Talib \& Juel (2006) show that each neutral curve exhibits only one mode of instability and we refer to this paper for a detailed parameter study of the different modes of instability encountered in this system. Multiple parametric-resonance modes are only found to arise for lower values of $W e$, $W e \lesssim 750$. Here however, we focus exclusively on the characterization of the FW in the limit of experimentally realizable parameter values.

The experimental set-up is presented in $\S 2$, followed by the description of the mathematical model and numerical analysis in $\S 3$. In $\S 4.1$, we compare experimental measurements of the onset of the FW and its critical wavenumber to the predictions of our viscous model. Excellent agreement between experiments and theory indicates that the simple model geometry is sufficient to accurately predict the onset of the FW instability over a wide range of viscosity ratios. The predictions of Lyubimov \& Cherepanov (1987) are compared with results from our viscous model in $\$ 4.2$. The viscous results tend toward their inviscid predictions only for very large frequencies of forcing. Interestingly, the model of Lyubimov \& Cherepanov (1987) consistently underestimates the threshold for fluids of equal viscosity, but generally overestimates the threshold for fluids of unequal viscosity. The effect of increasing the viscosity contrast is investigated numerically in $\S 4.3$. The viscosity ratio, $N_{1}$, is increased between 1 and $6 \times 10^{4}$, by raising the viscosity of the upper layer while keeping the viscosity of the lower layer constant. We identify four separate regions of $N_{1}$ where the system exhibits qualitatively different dynamics, which are reflected in the variation of the most unstable wavenumber with $N_{1}$ and correspond to either the stabilization or destabilization of the flow. The physical mechanisms responsible for the viscositydriven variations of the stability threshold are examined in $\S 4.4$, by focusing on the time-averaged perturbation streamfunctions. Conclusions are drawn in $\S 5$. 

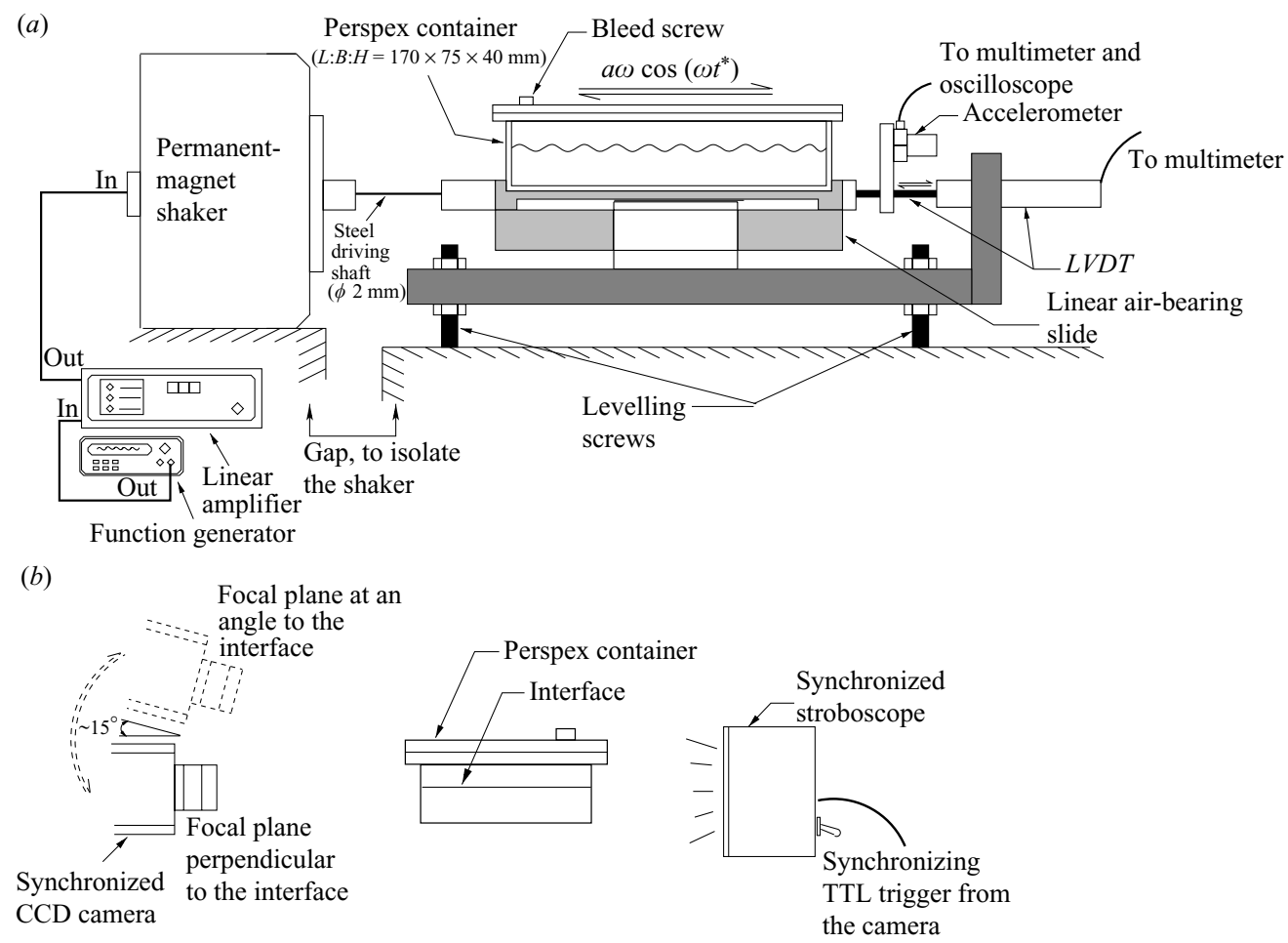

FIgURE 1. (a) Schematic side-view of the experimental apparatus. (b) Visualization set-up (viewed from the end).

\section{The experiment}

A schematic diagram of the experimental apparatus is shown in figure $1(a)$. The fluids were contained in a rectangular Perspex box of inner dimensions $170 \mathrm{~mm} \times$ $75 \mathrm{~mm} \times 40 \mathrm{~mm}$ and $4 \mathrm{~mm}$ thick walls, which was sealed with a Perspex lid and fitted with a bleed-hole to facilitate filling. The box was rigidly mounted on a linear, horizontal air-bearing slide (Nelson Air), which was supplied with compressed air. The slide was driven by a permanent-magnet shaker (LDS, V450) through a steel shaft with a diameter of $2 \mathrm{~mm}$. The shaker was powered by a linearly amplified, sinusoidal signal from a waveform generator (Agilent, 33220A). The shaker and oscillating set-up were mounted on separate tables, in order to isolate the liquids from the vibrational noise of the shaker.

The quality of the sinusoidal motion was monitored by an accelerometer mounted on the oscillating slide. The ratio of the power of the first harmonic and principal frequency peaks of the fast-Fourier-transformed accelerometer signal was found to decrease rapidly with increasing frequency. Thus, the harmonic content of the motion of the slide was less than $3 \%, 1.5 \%$ and $0.1 \%$ at frequencies of $f=5 \mathrm{~Hz}, f=20 \mathrm{~Hz}$ and $f>20 \mathrm{~Hz}$ respectively. The larger distortion of the sine wave at low frequencies was due to the mechanical limitations of the electromagnetic shaker. When setting up the experiment, the drive shaft was connected to the slide and carefully aligned with the shaker, so that lateral vibrations were minimized to an undetectable level, when monitored with a second accelerometer, mounted orthogonally to the direction of motion. 


$\begin{array}{ccc}\text { Lower layer } & v_{1}\left(\mathrm{~m}^{2} \mathrm{~s}^{-1}\right) & \rho_{1}\left(\mathrm{~kg} \mathrm{~m}^{-3}\right) \\ \text { Galden HT135 } & 1.12 \times 10^{-6} & 1752 \\ \text { Upper layer } & v_{2}\left(\mathrm{~m}^{2} \mathrm{~s}^{-1}\right) & \rho_{2}\left(\mathrm{~kg} \mathrm{~m}^{-3}\right) \\ \text { silicone oil }(100 \mathrm{cS}) & 1.14 \times 10^{-4} & 961 \\ \text { silicone oil }(200 \mathrm{cS}) & 2.10 \times 10^{-4} & 962 \\ \text { silicone oil }(1000 \mathrm{cS}) & 1.15 \times 10^{-3} & 966 \\ \text { silicone oil }(12500 \mathrm{cS}) & 1.35 \times 10^{-2} & 975\end{array}$

TABle 1. Physical properties of Galden HT135 and the silicone fluids at $18^{\circ} \mathrm{C}$, where $v_{\beta}$ denotes the kinematic viscosity and $\rho_{\beta}$ the density, in the lower layer $(\beta=1)$ and the upper layer $(\beta=2)$ respectively. The viscosity of the $12500 \mathrm{cS}$ silicone oil was not measured in the laboratory, and the value was taken from the data sheet at $18^{\circ} \mathrm{C}$.

Thus, the sinusoidal drive generated a linear displacement of the fluid-filled container about its rest position of $a \cos \left(2 \pi f t^{*}\right)$, where $f=\omega /(2 \pi)$ is the frequency of the displacement. The working frequency was in the range $10 \mathrm{~Hz}<f<60 \mathrm{~Hz}$, and the shaker could produce amplitudes of up to $a=9 \mathrm{~mm}$ at low frequencies. The dynamical displacement of the container was measured with a linearly variable differential transducer (LVDT, Solartron, Mach 1), whose signal enabled the feedback control of the amplitude of oscillation to better than $\pm 0.1 \%$.

The cell was filled with equal depths $\left(d_{1}=d_{2}=20 \mathrm{~mm}\right.$, where $d_{1}$ and $d_{2}$ are the depths of the lower and upper layers respectively) of Galden HT135 (a perfluoropolyether from Solvay Solexis) and silicone oil (polydimethylsiloxanes from Basildon Chemicals Ltd). This pair of immiscible fluids was chosen for its large density contrast and low interfacial tension. Also, the meniscus at the contact line with the Perspex vessel was found to be minimal. Different grades of silicone oil were used with kinematic viscosities between 100 and $12500 \mathrm{cS}$. The experiments were conducted in a temperaturecontrolled room at $18 \pm 1^{\circ} \mathrm{C}$, and the densities and dynamic viscosities of the fluids, measured at this temperature, are given in table 1 . Note that the density of silicone oils of different viscosity remains constant to within $1.5 \%$. The interfacial tension between Galden HT135 and silicone oil was measured nine times for each pair of fluids at $18^{\circ} \mathrm{C}$, using a Du Nouy ring, and found to be equal to $\gamma=6.8 \times 10^{-3} \mathrm{~N} \mathrm{~m}^{-1}$ for all of the grades of silicone oil investigated.

The FW arising in the experimental vessel was virtually independent of the transverse direction, and thus two-dimensional in nature. In the case of an upper layer of moderate viscosity, a considerable increase in the forcing parameters led to a transition to three-dimensional waves. In this paper, however, we focus on the primary onset of the FW.

The onset measurements were performed by setting the frequency and incrementing the amplitude in steps of $10 \mu \mathrm{m}$, leaving the flow to settle at each step for approximately 30 s, (i.e. between 300 and 1800 oscillations), and for more than $1000 \mathrm{~s}$ with the oil of largest viscosity of $12500 \mathrm{cS}$ (i.e. between $10^{4}$ and $4 \times 10^{4}$ oscillations). The onset of instability was detected using shadowgraphy. The interface was illuminated stroboscopically and synchronously with the drive frequency, and the deformation of the interface was captured with a synchronized, digital CCD camera, viewing the interface at an angle of $15^{\circ}$ from the horizontal, as illustrated in figure $1(b)$. The minimal deflection of the interface which could be detected was of $0.04 \mathrm{~mm}$. This value was estimated using deflectometry (Fermigier et al. 1992; Burgess et al. 2001), which consisted of imaging a fine grid through the fluid layers and measuring its distortion at onset due to 
(a)

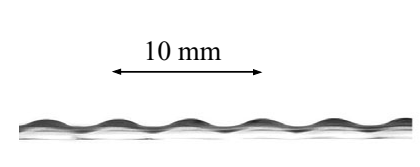

(b)

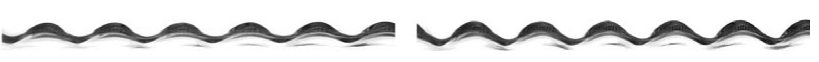

(d)

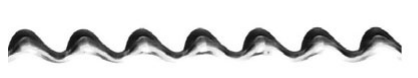

(e)

$(f)$

FIGURE 2. Frozen wave instability of the interface between silicone oil $\left(\rho_{2}=962 \mathrm{~kg} \mathrm{~m}^{-3}\right.$, $\left.\nu_{2}=2.10 \times 10^{-4} \mathrm{~m}^{2} \mathrm{~s}^{-1}\right)$ and Galden HT135 $\left(\rho_{1}=1752 \mathrm{~kg} \mathrm{~m}^{-3}, v_{2}=1.12 \times 10^{-6} \mathrm{~m}^{2} \mathrm{~s}^{-1}\right)$, under horizontal vibration. The frequency of forcing is $\omega=40 \pi \mathrm{rads}^{-1}$ and the amplitudes, $a$, are equal to: (a) $2.40 \mathrm{~mm},(b) 2.60 \mathrm{~mm},(c) 2.80 \mathrm{~mm},(d) 3.20 \mathrm{~mm},(e) 3.60 \mathrm{~mm},(f) 4.20 \mathrm{~mm}$. Note that snapshots $(a)-(c)$ and $(e)-(f)$ were captured at the zero-displacement position (i.e. zero acceleration), within the oscillatory cycle. Snapshot $(d)$, however, was taken slightly away from this neutral position, and thus, the waves appear slanted. All the waves are stationary in the oscillatory frame of reference.

interface deformation. The onset measurements were found to be increasingly difficult to resolve for increasing upper-layer viscosity, due to the reduction of the deflection of the interface following the increase of the wavelength of the instability. Thus, particular care had to be taken with $1000 \mathrm{cS}$ and $12500 \mathrm{cS}$ silicone oils. We nevertheless estimate the uncertainty in the onset measurements to be determined by the size of the amplitude step, and thus to be equal to $\pm 5 \mu \mathrm{m}$. The camera could also be aligned horizontally with the interface to record side-view snapshots of the growth of the FW. In figure 2, a series of snapshots, recorded for successive values of $a$, demonstrate the development of the interfacial instability for moderate values of the viscosity ratio $\left(N_{1}=187.5\right)$. The wavelength of the FW was measured at onset by determining the peak-to-peak distance, averaged over 5 to 8 waves within each image, and over five images. The errors in these measurements were calculated using t-statistics, as described by Beckwith, Marangoni \& Lienhard (1993), with a confidence level of $95 \%$.

\section{The model}

\subsection{Governing equations}

A schematic diagram of the model is shown in figure 3. We consider two superposed layers of incompressible and immiscible fluids bounded above and below by rigid plates, which are oscillating sinusoidally about their rest position with velocity $a \omega \cos \left(\omega t^{*}\right)$. The denser fluid is placed in the lower layer, so that the configuration is gravitationally stable. We choose a Cartesian coordinate system where the $x^{*}$-axis is parallel to the undeformed fluid interface, which lies at $z^{*}=0$, and the $z^{*}$-axis is parallel to the acceleration due to gravity. Each fluid layer is characterized by a density $\rho_{\beta}$, a kinematic viscosity, $v_{\beta}$ and a height, $d_{\beta}$, where the subscripts $\beta=1,2$ denote the lower and upper layers respectively. The interfacial tension between the two layers is denoted by $\gamma$.

Using $d_{2}, a \omega, \omega^{-1}$ and $\mu_{2} a \omega / d_{2}$, where $\mu_{2}=\rho_{2} v_{2}$ is the dynamic viscosity of the upper layer, as length, velocity, time and pressure scales respectively, the dimensionless Navier-Stokes equations governing the two-layer flow, in the frame of reference of 


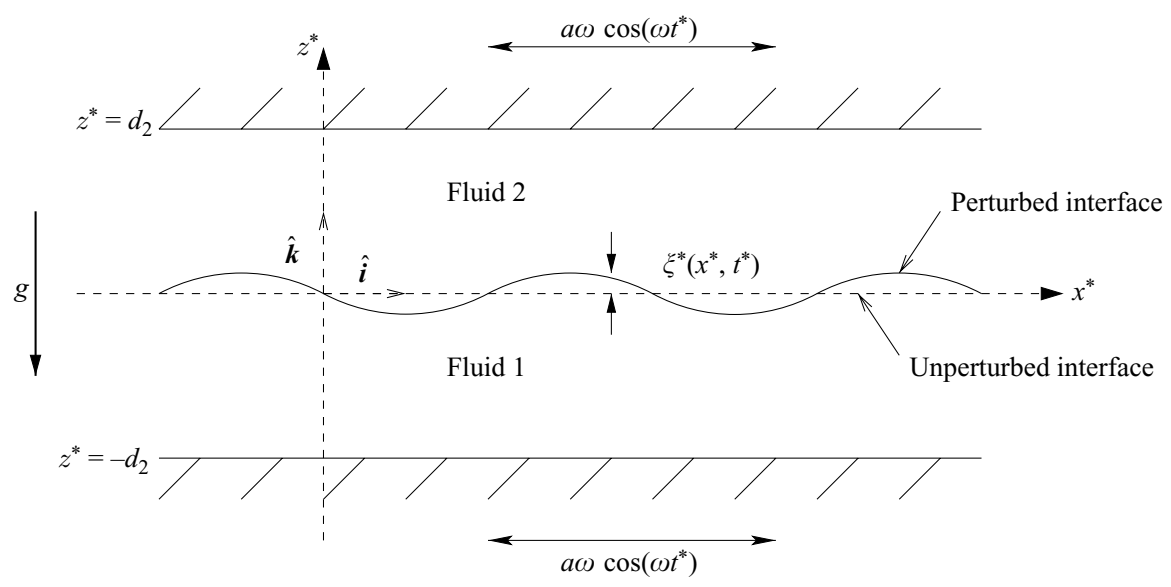

FIGURE 3. Schematic diagram of the two-layer fluid system. The unperturbed interface (horizontal dashed line) coincides with the $x^{*}$-axis and the perturbed interface is shown with a solid line. The superscript ${ }^{*}$ denotes dimensional variables.

the oscillating boundaries, become

$$
\begin{gathered}
\Omega \frac{\partial \boldsymbol{v}_{\beta}}{\partial t}+A \Omega\left(\boldsymbol{v}_{\beta} \cdot \nabla\right) \boldsymbol{v}_{\beta}=-R_{\beta} \nabla p_{\beta}+\frac{1}{N_{\beta}} \nabla^{2} \boldsymbol{v}_{\beta}-\frac{G_{0} \Omega}{A} \hat{\boldsymbol{k}}+\Omega \cos (t) \hat{\boldsymbol{i}}, \\
\nabla \cdot \boldsymbol{v}_{\beta}=0,
\end{gathered}
$$

where $\boldsymbol{v}_{\beta}=\left(u_{\beta}, w_{\beta}\right)$ is the velocity in each layer. $\Omega=\left(\omega d_{2}^{2}\right) / \nu_{2}$ and $A=a / d_{2}$ are the dimensionless frequency and amplitude of oscillation respectively, $R_{\beta}=\rho_{2} / \rho_{\beta}$ and $N_{\beta}=v_{2} / v_{\beta}$, where $R_{2}=N_{2}=1$, are the ratios of densities and viscosities respectively and $G_{0}=g /\left(d_{2} \omega^{2}\right)$ is the Froude number.

The rigid boundaries are stationary in the oscillatory frame of reference, and so the no-slip conditions are given by

$$
\boldsymbol{v}_{1}=0 \text { at } z=-d \text { and } \boldsymbol{v}_{2}=0 \quad \text { at } z=1,
$$

where $d=d_{1} / d_{2}=1$ is the ratio of layer depths. The primitive form of the interfacial conditions, comprising the kinematic condition, the continuity of velocity, and the balance of normal and tangential stresses respectively, at $z=\xi(x, t)$, where $\xi$ measures the interfacial deformation, are

$$
\begin{aligned}
\frac{1}{A} \frac{\partial \xi}{\partial t}+\left(\boldsymbol{v}_{1} \cdot \nabla\right) \xi & =\boldsymbol{v}_{1} \cdot \hat{\boldsymbol{k}}, \\
\boldsymbol{v}_{1} & =\boldsymbol{v}_{2}, \\
\left(\hat{\boldsymbol{n}} \cdot \pi_{1}\right) \cdot \hat{\boldsymbol{n}}-\left(\hat{\boldsymbol{n}} \cdot \pi_{2}\right) \cdot \hat{\boldsymbol{n}} & =\frac{\Omega}{W e A} \nabla \cdot \hat{\boldsymbol{n}}, \\
\left(\hat{\boldsymbol{t}} \cdot \pi_{1}\right) \cdot \hat{\boldsymbol{n}} & =\left(\hat{\boldsymbol{t}} \cdot \pi_{2}\right) \cdot \hat{\boldsymbol{n}} .
\end{aligned}
$$

$W e=\left(\rho_{2} d_{2}^{3} \omega^{2}\right) / \gamma$ is the Weber number, $\pi_{\beta}$ denotes the stress tensor in each fluid expressed as

$$
\pi_{\beta}=\frac{1}{R_{\beta} N_{\beta}}\left[\nabla \boldsymbol{v}_{\beta}+\left(\nabla \boldsymbol{v}_{\beta}\right)^{T}\right]-p_{\beta} \boldsymbol{I},
$$


where $\boldsymbol{I}$ is the identity matrix; $\hat{\boldsymbol{n}}$ is the outward, normal unit vector pointing from fluid 1 into fluid 2 and $\hat{\boldsymbol{t}}$ is the tangent, unit vector on the interface given by $\hat{\boldsymbol{n}}=\{-\partial \xi / \partial x, 1\}\left(1+(\partial \xi / \partial x)^{2}\right)^{-1 / 2}$, and $\hat{\boldsymbol{t}}=\{1, \partial \xi / \partial x\}\left(1+(\partial \xi / \partial x)^{2}\right)^{-1 / 2}$ respectively.

In addition, following Lyubimov \& Cherepanov (1987) and Khenner et al. (1999), we enforce a zero net volume flux in the $x$-direction,

$$
\int_{-d}^{0} \boldsymbol{v}_{1} \cdot \hat{\boldsymbol{i}} \mathrm{d} z+\int_{0}^{1} \boldsymbol{v}_{2} \cdot \hat{\boldsymbol{i}} \mathrm{d} z=0
$$

in order to model the counterflowing layers generated in vessels with endwalls.

\subsection{Base-flow solution}

The base flow, $\left(\overline{\boldsymbol{v}}_{\beta}, \bar{p}_{\beta}\right)$, is periodic, parallel to the horizontal boundaries and the interface remains unperturbed. By enforcing condition (3.8), we impose a non-zero streamwise pressure gradient,

$$
\frac{\partial \bar{p}_{\beta}}{\partial z}=S_{\beta} \mathrm{e}^{\mathrm{i} t},
$$

where $S_{1}=S_{2}=S$, since the normal stress condition when the interface is unperturbed and flow is in the $x$-direction only, states that $\bar{p}_{1}=\bar{p}_{2}$. Thus, we induce a base flow where the two fluid layers move in opposite directions, which takes the form

$$
\bar{u}_{\beta}(z, t)=\operatorname{Re}\left[\mathrm{e}^{\mathrm{i} t}\left(A_{\beta} \mathrm{e}^{m_{\beta} z}+\mathrm{i}\left(\frac{S R_{\beta}}{\Omega}-1\right)+B_{\beta} \mathrm{e}^{-m_{\beta} z}\right)\right],
$$

and

$$
\bar{p}_{\beta}(x, z, t)=-\frac{G_{0} \Omega}{R_{\beta} A} z+S x \mathrm{e}^{\mathrm{i} t}+C,
$$

where $m_{\beta}=\sqrt{\mathrm{i} \Omega N_{\beta}}$ with $\mathrm{i}=(-1)^{1 / 2}$, the integration constants $A_{\beta}, B_{\beta}$ and $S$ are determined by imposing conditions (3.3), (3.5), (3.7) and (3.8), and $C$ is an arbitrary constant. The values of the constants were determined with the symbolic toolbox in MATLAB. Base-flow solutions, plotted at $t=0$ and $t=\pi / 2$ for five values of the viscosity ratio, $N_{1}$, are shown in figure 4.

\subsection{Perturbation equations}

According to Squire's theorem, which was extended to two layers by Hesla, Pranckh \& Preziosi (1986), it is sufficient to consider only two-dimensional disturbances to the base flow. This choice is also justified by the experimental observation of a two-dimensional FW. In deriving the stability equations, we express the governing equations (3.1)-(3.2) in terms of a streamfunction, $\psi_{\beta}$, defined by

$$
u_{\beta}=\frac{\partial \psi_{\beta}}{\partial z}, \quad w_{\beta}=-\frac{\partial \psi_{\beta}}{\partial x},
$$

and seek a normal-mode solution (Drazin \& Reid 1981) of the infinitesimally perturbed base flow of the form

$$
\left(\psi_{\beta}, p_{\beta}, \xi\right)=\left(\bar{\psi}_{\beta}, \bar{p}_{\beta}, 0\right)+\left[\phi_{\beta}(z, t), P_{\beta}(z, t), h(t)\right] \mathrm{e}^{\mathrm{i} k x}+\text { c.c. },
$$

where $k=2 \pi d_{2} / \lambda^{*}$ is the dimensionless wavenumber of the disturbance, $\lambda^{*}$ is the dimensional wavelength, and c.c. denotes the complex conjugate. By substituting (3.11) into the Navier-Stokes equations (3.1), as well as the boundary and interfacial conditions (3.3)-(3.8), subtracting out the base state, eliminating the pressure term, and neglecting higher-order terms, we obtain the following Orr-Sommerfeld equations 

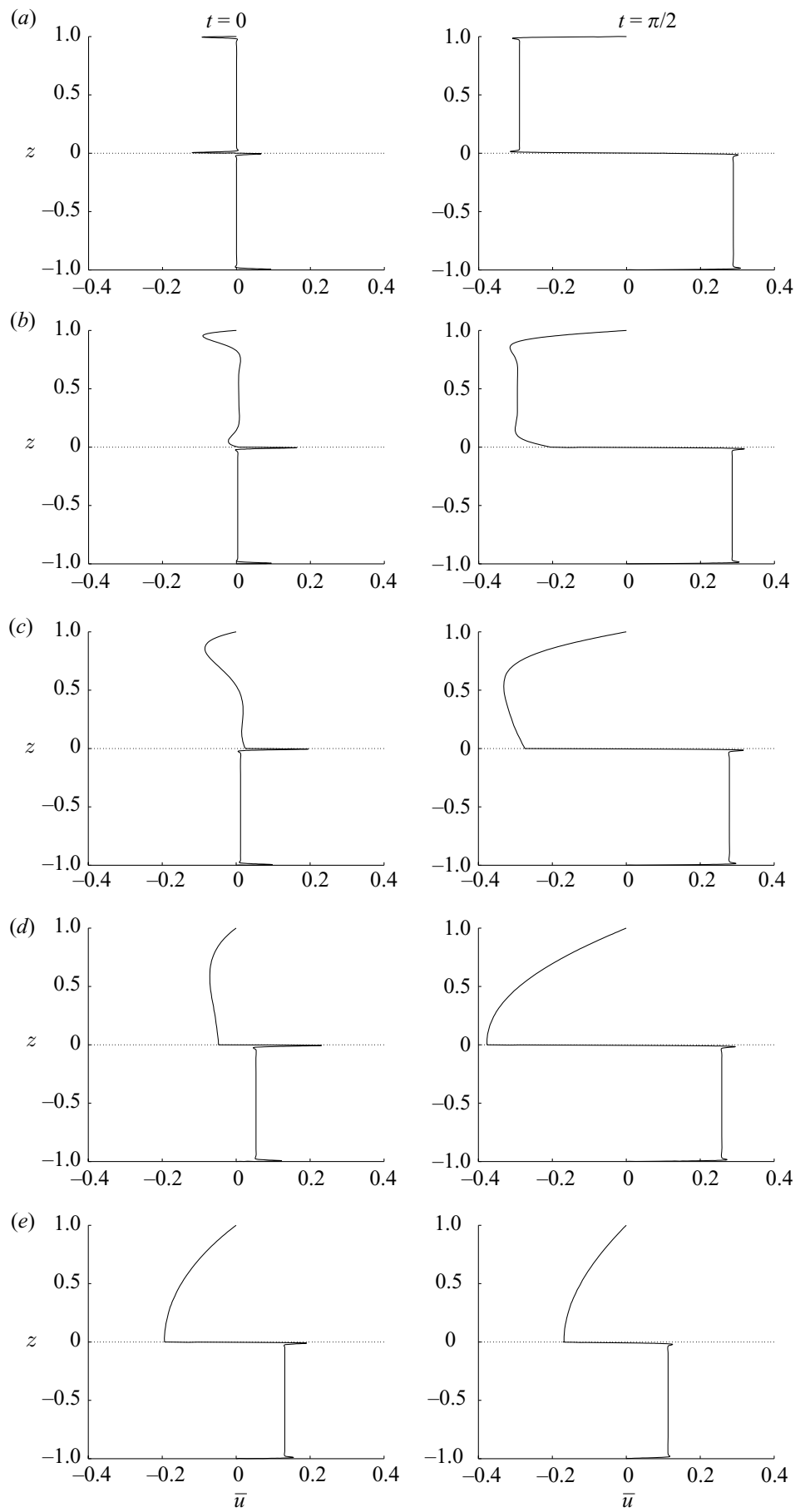

FIGURE 4. Base-flow solutions calculated for parameter values associated with Galden HT135 and silicone oils of different viscosities, are shown at the time intervals, $t=0$ and $t=\pi / 2$, covering a quarter of a period of oscillation. Snapshots at $t=3 \pi / 2, t=\pi$ and at regular time intervals within the second half of the oscillation cycle can be obtained by suitable reflections of these images. The non-dimensional parameters are $R_{1}=0.55, A=0.09, G_{0}=3.11 \times 10^{-2}$, $W e=1.74 \times 10^{4}$ and $d=1:$ (a) $N_{1}=1, \Omega=5.03 \times 10^{4}$; (b) $N_{1}=1 \times 10^{2}, \Omega=5.03 \times 10^{2} ;(c)$ $N_{1}=1 \times 10^{3}, \Omega=5.03 \times 10^{1} ;(d) N_{1}=1 \times 10^{4}, \Omega=5.03 ;$ and $(e) N_{1}=6 \times 10^{4}, \Omega=8.38 \times 10^{-1}$. Note the thinness of the boundary layers in the lower layer where the non-dimensional frequency is $\Omega_{1}=\Omega N_{1}=5.03 \times 10^{4}$. 
for the two-layer flow:

$$
\left(\Omega \frac{\partial}{\partial_{t}}+\mathrm{i} k A \Omega \bar{u}_{\beta}\right)\left(\phi_{\beta}^{\prime \prime}-k^{2} \phi_{\beta}\right)-\mathrm{i} k A \Omega \bar{u}_{\beta}^{\prime \prime} \phi_{\beta}+\frac{1}{N_{\beta}}\left(2 k^{2} \phi_{\beta}^{\prime \prime}-k^{4} \phi_{\beta}-\phi_{\beta}^{\prime \prime \prime \prime}\right)=0,
$$

where the prime denotes partial differentiation with respect to $z$. The no-slip boundary conditions become

$$
\phi_{1}=0, \quad \phi_{1}^{\prime}=0 \quad \text { at } z=-d \quad \text { and } \quad \phi_{2}=0, \quad \phi_{2}^{\prime}=0 \quad \text { at } z=1,
$$

and the linearized interfacial conditions, with $\phi$ expanded in a Taylor series about $z=0$, become

$$
\begin{aligned}
& \frac{1}{A} \frac{\partial h}{\partial t}+\mathrm{i} k \bar{u}_{1} h+\mathrm{i} k \phi_{1}=0, \quad \text { kinematic condition, } \\
& \phi_{1}^{\prime}-\phi_{2}^{\prime}+h\left(\bar{u}_{1}^{\prime}-\bar{u}_{2}^{\prime}\right)=0, \quad \text { continuity of velocity, } \\
& \phi_{1}-\phi_{2}=0, \quad \text { continuity of velocity, } \\
& {\left[\frac{\Omega}{R_{1}} \frac{\partial \phi_{1}^{\prime}}{\partial t}-\frac{\mathrm{i} k A \Omega}{R_{1}} \bar{u}_{1}^{\prime} \phi_{1}+\frac{\mathrm{i} k A \Omega}{R_{1}} \bar{u}_{1} \phi_{1}^{\prime}+\frac{3 k^{2}}{N_{1} R_{1}} \phi_{1}^{\prime}-\frac{1}{N_{1} R_{1}} \phi_{1}^{\prime \prime \prime}+\frac{\mathrm{i} k G_{0} \Omega}{A R_{1}} h\right]} \\
& -\left[\Omega \frac{\partial \phi_{2}^{\prime}}{\partial t}-\mathrm{i} k A \Omega \bar{u}_{2}^{\prime} \phi_{2}+\mathrm{i} k A \Omega \bar{u}_{2} \phi_{2}^{\prime}+3 k^{2} \phi_{2}^{\prime}-\phi_{2}^{\prime \prime \prime}+\frac{\mathrm{i} k G_{0} \Omega}{A} h\right] \\
& +\frac{\mathrm{i} k^{3} \Omega}{W e A} h=0, \quad \text { normal stress balance, } \\
& \phi_{1}^{\prime \prime}+h \bar{u}_{1}^{\prime \prime}+k^{2} \phi_{1}-N_{1} R_{1}\left(\phi_{2}^{\prime \prime}+h \bar{u}_{2}^{\prime \prime}+k^{2} \phi_{2}\right)=0, \quad \text { tangential stress balance. }
\end{aligned}
$$

\subsection{Numerical solution}

A detailed description of the numerical method can be found in Talib \& Juel (2006). Therefore we only recall essential details here. A linear transformation was used to map the physical coordinates, $z \in[-d, 0]$ in the lower layer, and $z \in[0,1]$ in the upper layer, each onto $\zeta \in[-1,1]$, so that the equations (3.12)-(3.18) could be discretized with Gauss-Lobatto-Chebyshev collocation points, in order to maximize resolution near the boundaries and the interface. The solution was expanded as a Lagrange polynomial series, truncated at the $L_{\beta}$ th term, at the collocation points $\zeta_{i}$, so that

$$
\phi_{\beta}\left(\zeta_{i}, t\right)=\sum_{j=0}^{L_{\beta}} \phi_{\beta j}(t) C_{j}\left(\zeta_{i}\right), \quad i=0,1, \ldots, L_{\beta},
$$

where $\phi_{\beta j}(t)$ are the unknown time-dependent amplitudes to be determined and $C_{j}\left(\zeta_{i}\right)$ are the Lagrange basis functions, associated with the Gauss-Lobatto-Chebyshev collocation points, and given by

$$
C_{j}\left(\zeta_{i}\right)=\frac{(-1)^{j}\left(\zeta_{i}^{2}-1\right) T_{L_{\beta}}^{\prime}\left(\zeta_{i}\right)}{\bar{c}_{j} L_{\beta}^{2}\left(\zeta_{i}-\zeta_{j}\right)}, \quad i, j=0,1, \ldots, L_{\beta},
$$

where $\bar{c}_{0}=\bar{c}_{L_{\beta}}=2, \bar{c}_{1}=\ldots=\bar{c}_{L_{\beta}-1}=1$ and $T_{L_{\beta}}^{\prime}$ is the first derivative of the $L_{\beta}$ th-order Chebyshev polynomial. The first-order derivative matrix was determined explicitly by differentiating the Lagrange polynomials defined in equation (3.20). Higher-order derivatives were expressed as multiple powers of the first-order derivative matrix.

Following Lanczos' (1956) method, the governing equations, (3.12), were evaluated at the interior collocation points and the boundary conditions (3.13)-(3.18) at the 
boundary collocation points $\zeta=1$ and $\zeta=-1$, yielding a $\left(L_{1}+L_{2}+3\right) \times\left(L_{1}+L_{2}+3\right)$ algebraic equation of the form

$$
\boldsymbol{B} \frac{\partial \boldsymbol{x}}{\partial t}=\left(\boldsymbol{M}_{0}+\boldsymbol{M}(t)\right) \boldsymbol{x},
$$

where $\boldsymbol{x}$ is the unknown vector

$$
\boldsymbol{x}(t)=\left(\phi_{10}, \phi_{11}, \ldots, \phi_{1 L_{1}}, \phi_{20}, \phi_{21}, \ldots, \phi_{2 L_{2}}, h\right)^{T}(t),
$$

$\boldsymbol{M}(t)=\boldsymbol{M}_{\mathrm{s}} \sin (t)+\boldsymbol{M}_{\mathrm{c}} \cos (t)$ is a $2 \pi$-periodic matrix, and $\boldsymbol{B}$ and $\boldsymbol{M}_{0}$ are constantcoefficient matrices.

The time-dependent system (3.21) was solved using a fast iterative scheme based upon the Newton-Raphson method that was developed by Or (1997) for a viscous single layer under oscillating shear. According to Floquet theory (Nayfeh \& Mook 1979), the solution of (3.21) is of the form

$$
\boldsymbol{x}(t)=Z(t) \exp (\sigma t),
$$

where $Z(t)$ is a $2 \pi$-periodic vector function and $\sigma$ is the Floquet exponent. $Z(t)$ is expanded as a complex Fourier series, truncated at order $K$, so that

$$
\boldsymbol{x}(t)=\sum_{n=-K}^{K} \boldsymbol{x}_{n} \mathrm{e}^{\mathrm{i} n t+\sigma t},
$$

where $\sigma=\sigma_{r}+\mathrm{i} \sigma_{i}$ and $\boldsymbol{x}_{n}$ are constant vector coefficients, and substituted into the algebraic equation (3.21). We focus on the harmonic solution with $\sigma_{i}=0$, which is found experimentally. Furthermore, neither Or (1997) nor Khenner et al. (1999) detected any subharmonic solution, due to the time-symmetry of the base flow as discussed by Or (1997). By substitution of the solution (3.23) into equation (3.21) and employing Or's (1997) iterative procedure, a polynomial eigenvalue equation of order $K+1$ is obtained for $\boldsymbol{x}_{0}$. We evaluate the largest real eigenvalue (recall $\sigma_{i}=0$ ) for which the determinant vanishes for a given set of parameter values, and compute the marginal stability of the two-layer flow by iterating on the value of the amplitude parameter, $A$, starting with $A=0.01$.

All the numerical computations were carried out in MATLAB. Each linear stability result was checked for convergence by consecutively increasing each of the Fourier and Chebyshev modes in the lower and upper layers by two. Beyond the converged iterate, increasing the number of Fourier and Chebyshev modes did not affect the first four digits of the critical amplitude, $A_{c}$. The number of Chebyshev modes required for a converged solution increased with the non-dimensional frequency in each layer, $\Omega_{2}=\Omega$ and $\Omega_{1}=\Omega N_{1}$, because the boundary layer thicknesses are reduced. The effect of the non-dimensional frequencies on the thickness of the boundary layers of the base flow solution is illustrated in figure 4, where $\Omega_{2}=3.72$ and $\Omega_{1}=4.48 \times 10^{4}$. The spacing of the Gauss-Lobatto-Chebyshev collocation points near the boundaries is $O\left(L_{\beta}^{-2}\right)$, and it was found that at least one collocation point had to lie within the boundary layer to ensure the convergence of the solution. The results presented in this paper were calculated for $82<L_{1}<110,30<L_{2}<90$ and $K=14$. The code was validated by successfully reproducing several known results for both single-layer and two-layer oscillatory flows. The stability results obtained using the Chebyshev collocation code were found to be in excellent agreement with those of Khenner et al. (1999) in the limit of large viscosity $(\Omega<360)$. We were also able 


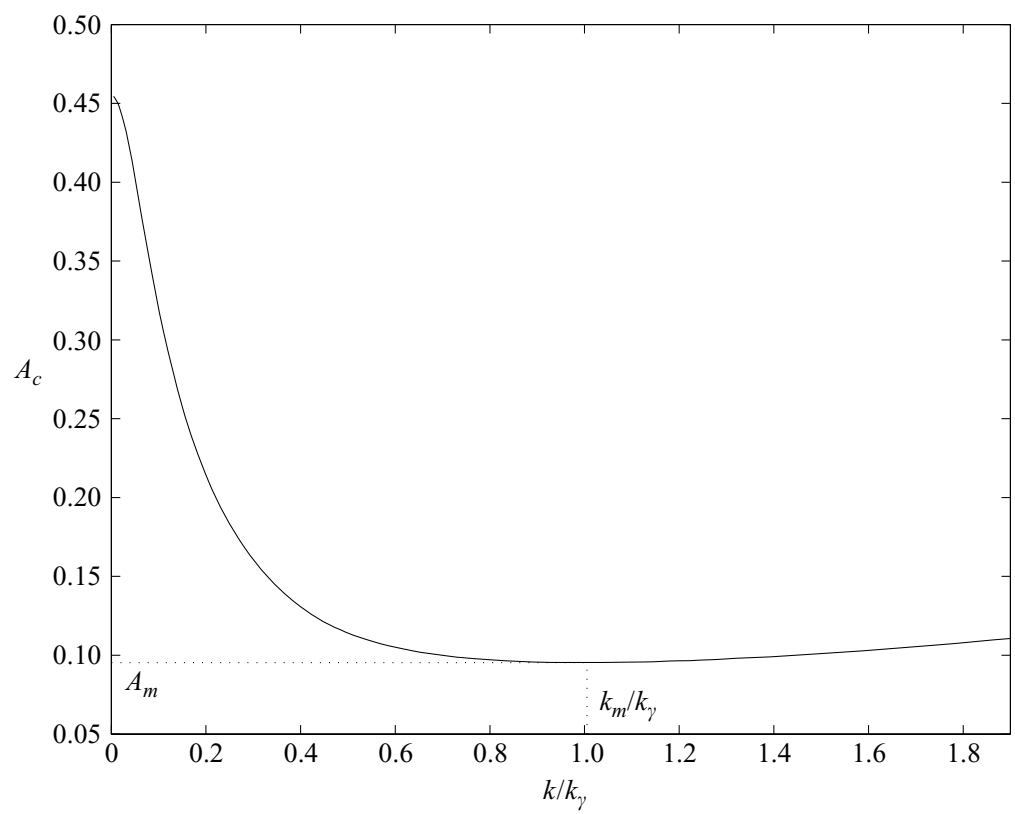

FIGURE 5. Marginal stability curve in terms of the critical amplitude $A_{c}$ versus the wavenumber $k / k_{\gamma}$, where $k_{\gamma}=d_{2} / l_{c}$ is the capillary wavenumber. The parameters are calculated for the material properties of Galden HT135 and $1000 \mathrm{cS}$ silicone given in table 1, a forcing frequency of $\omega=40 \pi \mathrm{rad} \mathrm{s}^{-1}$ and layer depths of $d_{1}=d_{2}=2 \times 10^{-2} \mathrm{~m}$. Thus, the non-dimensional parameters are $\Omega=3.72, N_{1}=1.03 \times 10^{3}, R_{1}=0.55, G_{0}=3.11 \times 10^{-2}, W e=1.74 \times 10^{4}$ and $d=1 . A_{m}$ is the critical amplitude of the most unstable wavenumber, $k_{m}$.

to reproduce Or's (1997) results by setting the upper-layer flow parameters to those of air $\left(v_{2}=15.11 \times 10^{-6} \mathrm{~m}^{2} \mathrm{~s}^{-1}\right.$ and $\left.\rho_{2}=1.29 \mathrm{~kg} \mathrm{~m}^{-3}\right)$, and omitting condition (3.8).

An example of a marginal stability curve, calculated using the properties of Galden HT135 and $1000 \mathrm{cS}$ silicone oil for $\omega=40 \pi \mathrm{rad} \mathrm{s}^{-1}$, is shown in figure 5. This curve exhibits only one finite-wavelength mode and remains qualitatively similar over the range of parameters explored in this paper. The minimum of the curve yields the critical value that should be compared with the experimental measurements, as it corresponds to the critical amplitude, $A_{m}$, of the most unstable wavenumber, $k_{m}$. Note that the broad minimum of the marginal stability curve implies that there is a range of wavenumbers with similar stability characteristics. Given that the resolution of the simulations is limited to four digits, we often find several neighbouring values of $k$ corresponding to the value of $A_{m}$, and thus estimate $k_{m}$ to be the average of these values.

For each value of $\left(A_{m}, k_{m}\right)$, the perturbation streamfunction could be calculated by computing the eigenfunction associated with the leading eigenvalue calculated at the point of marginal stability. The perturbation streamfunction was subsequently reconstructed in physical space and time-averaged over one period of forcing to yield the curves presented in $\S 4.4$.

\section{Results}

\subsection{Comparison between experiments and linear stability calculations}

We present the critical amplitude of oscillation in figure $6(a)$ and the critical wavenumber in figure $6(b)$, as a function of the dimensional forcing frequency. We choose 

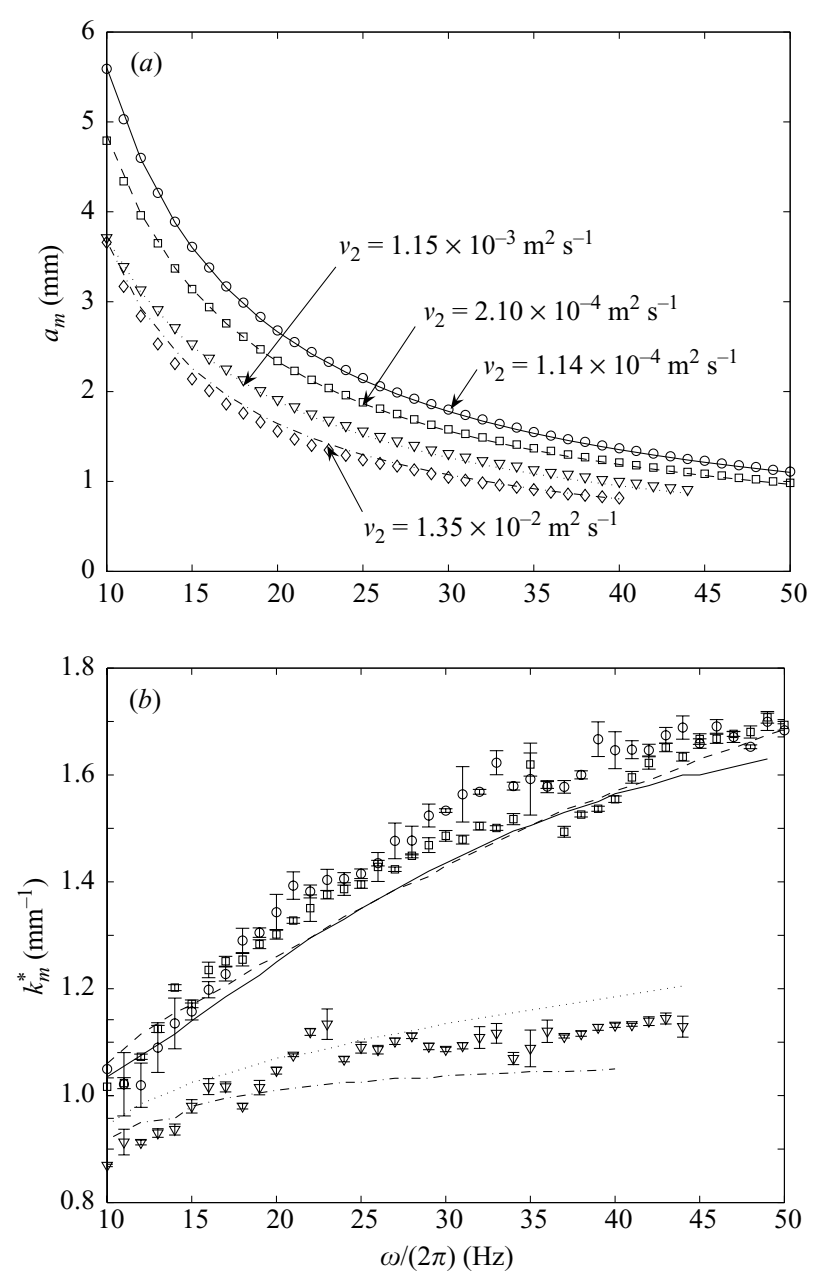

FIGURE 6. Onset of the frozen wave instability: comparison between experimental (symbols) and numerical (lines) results: $(a)$ dimensional critical amplitude, $a_{m}$, versus frequency of forcing; (b) dimensional most unstable wavenumber, $k_{m}^{*}$, versus frequency of forcing. The lower-layer viscosity is $v_{1}=1.12 \times 10^{-6} \mathrm{~m}^{2} \mathrm{~s}^{-1}$ and the upper-layer viscosity is: $v_{2}=1.14 \times 10^{-4} \mathrm{~m}^{2} \mathrm{~s}^{-1}$ $(\bigcirc,-) ; v_{2}=2.10 \times 10^{-4} \mathrm{~m}^{2} \mathrm{~s}^{-1}(\square,--) ; v_{2}=1.15 \times 10^{-3} \mathrm{~m}^{2} \mathrm{~s}^{-1}(\nabla, \cdots)$; and $\nu_{2}=1.35 \times$ $10^{-2} \mathrm{~m}^{2} \mathrm{~s}^{-1}(\diamond,-\cdot-)$. The other material properties are given in section 2 .

to display the comparison in dimensional form, $a_{m}$ and $k_{m}^{*}$ versus $\omega$, since a change in forcing frequency $\omega$ alters not only the non-dimensional frequency $\Omega$, but also $W e$ and $G_{0}$. In addition, the wide range of $\nu_{2}$ examined would make it difficult to compare the results on the same graph, because $\Omega$ varies with $\nu_{2}$. The experimental data are plotted with symbols and the numerical results with lines. The results include four different values of the viscosity contrast, achieved by using the fluids listed in table 1 .

The results shown in figure 6(a) demonstrate excellent quantitative agreement between the experiments and the stability calculations for all the fluid parameters investigated. Quantitative agreement is also achieved in the wavenumber comparison shown in figure $6(b)$, although the experimental measurements exhibit increasing scatter as the viscosity of the upper layer is increased. In particular, we were not able to accurately extract an experimental critical wavenumber, when using the most 
viscous silicone oil $(12500 \mathrm{cS})$. In this case, finite-amplitude oil fingers were observed to grow into the layer of Galden HT135 at the experimental onset value, so that the bifurcation leading to onset could not be resolved. Our results suggest that the range of amplitudes for which sinusoidal waves are observed decreases sharply with the increase of $\nu_{2}$, and that it is less than the amplitude step of $10 \mu \mathrm{m}$ in the case of $12500 \mathrm{cS}$ silicone oil.

The results of figure 6 indicate that our stability model, derived for oscillating, counterflowing layers of infinite lateral extent, is sufficient to predict the experimental onset of the FW quantitatively. The quality of the agreement implies that the endwalls of the experimental vessel only affect the flow and the shape of the interface locally. The experimental wavelength is of the order of $5 \mathrm{~mm}$, which is small compared with the length of the vessel of $l=170 \mathrm{~mm}$. Furthermore, the instability threshold and critical wavenumber remained unchanged to within less than $2.5 \%$, when experiments were performed in a vessel which was shorter by $30 \%(l=110 \mathrm{~mm})$. The visualization of the flow, using $57 \mu \mathrm{m}$ polyamide beads in the oil, indicated the presence of regions of recirculation within the more viscous layer, which were generated by sloshing. These recirculations, however, appeared to be localized within approximately $2 \mathrm{~cm}$ of the endwalls, and did not affect the flow in the central part of the vessel. Although further weak recirculations may have been present, which were not detected on the time scale of the experiments, the results of figure 6 suggest that they did not significantly affect the onset of the FW.

It is clear from figure $6(a)$ that increasing the viscosity contrast between the layers systematically lowers the stability threshold, and thus acts to destabilize the flow. This effect is particularly pronounced for the $100 \mathrm{cS}, 200 \mathrm{cS}$ and $1000 \mathrm{cS}$ silicone fluids, whereas the gap between the curves corresponding to the $1000 \mathrm{cS}$ and $12500 \mathrm{cS}$ silicone fluids is relatively smaller despite a tenfold increase in $v_{2}$. The destabilizing effect of the viscosity contrast observed in figure $6(a)$ is reminiscent of the result of the viscous, steady Kelvin-Helmholtz analysis of Barnea \& Taitel (1993). Given the excellent agreement between experiment and theory, we choose to perform a detailed numerical investigation of the effect of viscosity contrast on the stability of the flow, which is discussed in $\S 4.3$.

As for the stability threshold, the most unstable wavenumber shown in figure $6(b)$ exhibits a significant reduction when the viscosity contrast is increased, with the most substantial change observed between the curves corresponding to $200 \mathrm{cS}$ and $1000 \mathrm{cS}$ silicone oils, and is given in $\S 4.3$ a more complete discussion.

\subsection{Comparison with the inviscid theory of Lyubimov \& Cherepanov (1987)}

The results of $\S 4.1$ clearly demonstrate that a viscous model is necessary to quantitatively predict the experimental stability thresholds. Measurements of the onset of the FW by Ivanova et al. (2001) with $N_{1}=93.5$ and of its wavelength near onset by Wunenburger et al. (1999) with $N_{1} \simeq 1$ were found to be in qualitative agreement with the inviscid model of Lyubimov \& Cherepanov (1987), valid in the limit of large frequencies and vanishing amplitudes of forcing. A comparison is shown in figures 7 and 8 between the stability results of our viscous model and the inviscid theory. The stability results are presented in figure 7 in terms of the vibrational parameter $W_{m}=A_{m}^{2} / G_{0}$ introduced by Ivanova et al. (2001). $W_{m}$ measures the forcing acceleration relative to the acceleration due to gravity, so that the inviscid model yields a constant threshold depicted with a solid line. The most unstable wavenumber $k_{m}$ is shown in figure 8 relative to the capillary wavenumber $k_{\gamma}$. We present viscous stability calculations for $N_{1}=1$ in part $(a)$ of figures 7 and $8\left(v_{1}=v_{2}=3 \times 10^{-6} \mathrm{~m}^{2} \mathrm{~s}^{-1}\right)$ 

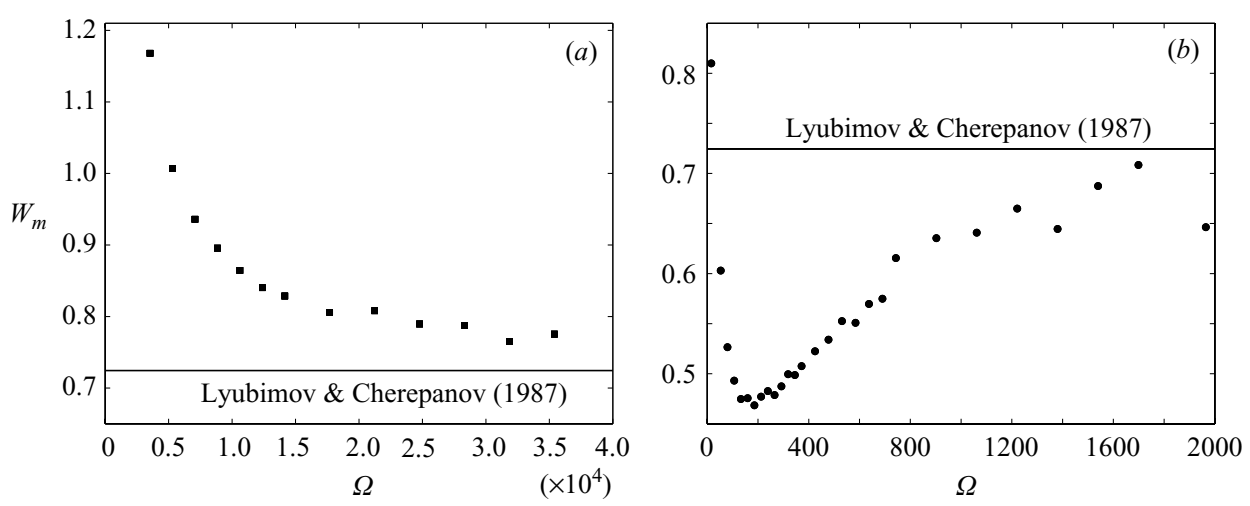

FIGURE 7. Comparison between the viscous two-layer model and the inviscid, high-frequency predictions of Lyubimov \& Cherepanov (1987) in terms of $W_{m}=A_{m}^{2} / G_{0}$ versus $\Omega$ for different values of the viscosity ratio: $(a) N_{1}=1 \quad\left(v_{1}=v_{2}=3 \times 10^{-6} \mathrm{~m}^{2} \mathrm{~s}^{-1}\right) ;(b) N_{1}=100 \quad\left(v_{1}=2 \times\right.$ $10^{-6} \mathrm{~m}^{2} \mathrm{~s}^{-1}$ and $v_{2}=2 \times 10^{-4} \mathrm{~m}^{2} \mathrm{~s}^{-1}$ ). The material properties are $\rho_{1}=1973 \mathrm{~kg} \mathrm{~m}^{-3}, \rho_{2}=$ $967 \mathrm{~kg} \mathrm{~m}^{-3}, \gamma=5.0 \times 10^{-3} \mathrm{~N} \mathrm{~m}^{-1}$ and $d_{1}=d_{2}=1.3 \times 10^{-2} \mathrm{~m}$. The inviscid model systematically underestimates the stability threshold when $N_{1}=1$, but generally overestimates the threshold when $N_{1}=100$.
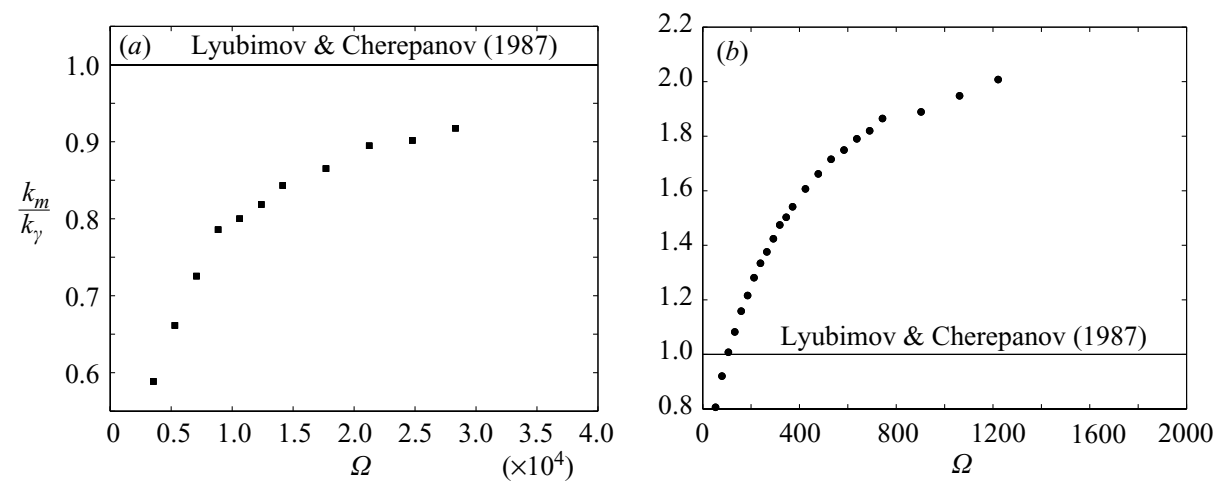

FIGURE 8. Comparison between the viscous two-layer model and the inviscid, high-frequency predictions of Lyubimov \& Cherepanov (1987) in terms of the most unstable wavenumber (relative to the capillary wavenumber), $k / k_{\gamma}$, versus $\Omega$ for different values of the viscosity ratio: (a) $N_{1}=1 \quad\left(v_{1}=v_{2}=3 \times 10^{-6} \mathrm{~m}^{2} \mathrm{~s}^{-1}\right) ;(b) N_{1}=100\left(v_{1}=2 \times 10^{-6} \mathrm{~m}^{2} \mathrm{~s}^{-1}\right.$ and $v_{2}=2 \times$ $\left.10^{-4} \mathrm{~m}^{2} \mathrm{~s}^{-1}\right)$. The material properties are $\rho_{1}=1973 \mathrm{~kg} \mathrm{~m}^{-3}, \rho_{2}=967 \mathrm{~kg} \mathrm{~m}^{-3}, \gamma=5.0 \times$ $10^{-3} \mathrm{~N} \mathrm{~m}^{-1}$ and $d_{1}=d_{2}=1.3 \times 10^{-2} \mathrm{~m}$. When $N_{1}=1, k / k_{\gamma}$ tends to 1 in the limit of large $\Omega$. When $N_{1}=100$, however, $k / k_{\gamma}$ far exceeds 1 in the limit of large $\Omega$.

and $N_{1}=100$ in part $(b)\left(v_{1}=2 \times 10^{-6} \mathrm{~m}^{2} \mathrm{~s}^{-1}\right.$ and $\left.v_{2}=2 \times 10^{-4} \mathrm{~m}^{2} \mathrm{~s}^{-1}\right)$. Note that successive calculations were performed with increasing values of $\omega$ as in the experiment, yielding not only increased dimensionless frequencies, $\Omega$, but also changes in the parameters $G_{0}$ and $W e$. For simplicity, however, we choose to show $W_{m}$ and $k_{m} / k_{\gamma}$ as functions of $\Omega$ only.

In the limit of large $\Omega$, both sets of viscous calculations yield values of $W_{m}$ that tend toward the threshold of Lyubimov \& Cherepanov (1987). The dimensional frequencies of forcing necessary to achieve good agreement with the inviscid model when $N_{1}=100\left(\omega \gtrsim 260 \mathrm{rad} \mathrm{s}^{-1}\right)$ are considerably larger than when $N_{1}=1\left(\omega \gtrsim 60 \mathrm{rad} \mathrm{s}^{-1}\right)$. The scatter in the numerical data for large $\Omega$ is linked to the choice of the vibrational 

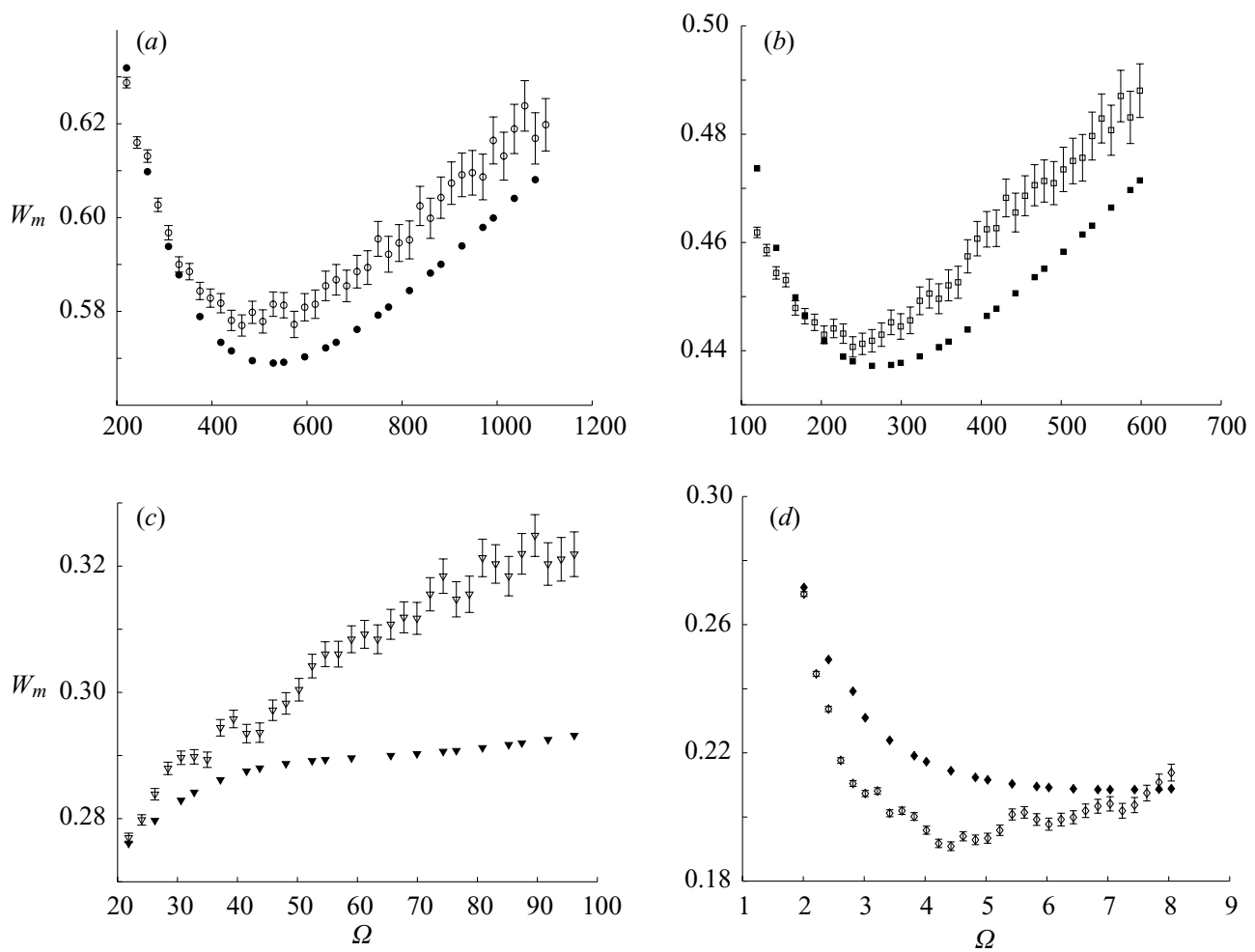

FiguRE 9. Experimental (open symbols) and numerical (solid symbols) onset values from figure 6 plotted in terms of $W_{m}$, the critical dimensionless acceleration corresponding to the most unstable wavenumber, versus $\Omega$ for silicone oil: (a) $100 \mathrm{cS} ;(b) 200 \mathrm{cS} ;(c) 1000 \mathrm{cS} ;(d) 12500 \mathrm{cS}$. The inviscid model of Lyubimov \& Cherepanov (1987) predicts $W_{m}=0.71$.

parameter $W_{m}$, which is not only proportional to $a_{m}^{2}$ but also to $\omega^{2}$. Hence, the small numerical uncertainty arising from the finite four-digit resolution of the calculations is significantly amplified at large values of $\Omega$. $W_{m}$ calculated for $N_{1}=1$ decreases monotonically with $\Omega$, whereas for $N_{1}=100$, the data exhibit a sharp decrease at low $\Omega$ and a subsequent increase toward the threshold of Lyubimov \& Cherepanov (1987) when $\Omega>200$. Hence, the inviscid model systematically underestimates the stability threshold in the case of fluids of equal viscosity, whereas the stability of two layers of unequal viscosity is generally overestimated.

It can be seen in figure 8 that when $N_{1}=1, k_{m}$ tends toward the capillary wavenumber, $k_{\gamma}=18.3$, predicted by Lyubimov \& Cherepanov (1987) as $\Omega$ increases. When $N_{1}=100, k_{m}$ also increases monotonically but grows far beyond the value of $k_{\gamma}$. This result is confirmed experimentally with the data presented in dimensional form in figure $6(b)$. At given $\Omega$, i.e. in dimensionless form, both experimental and numerical results indicate that $k_{m}$ increases with viscosity contrast. Thus, the wavenumber appears to be strongly modified by the presence of viscous forces, when there is a significant viscosity contrast between the layers.

A comparison between experimental and numerical results expressed in terms of $W_{m}$ rather than critical amplitude is a more severe test of the model than the comparison presented in figure 6, and is shown in figure 9 for all four sets of data. The theory of Lyubimov \& Cherepanov (1987) predicts $W_{m}=0.71$ for the fluids used in the 


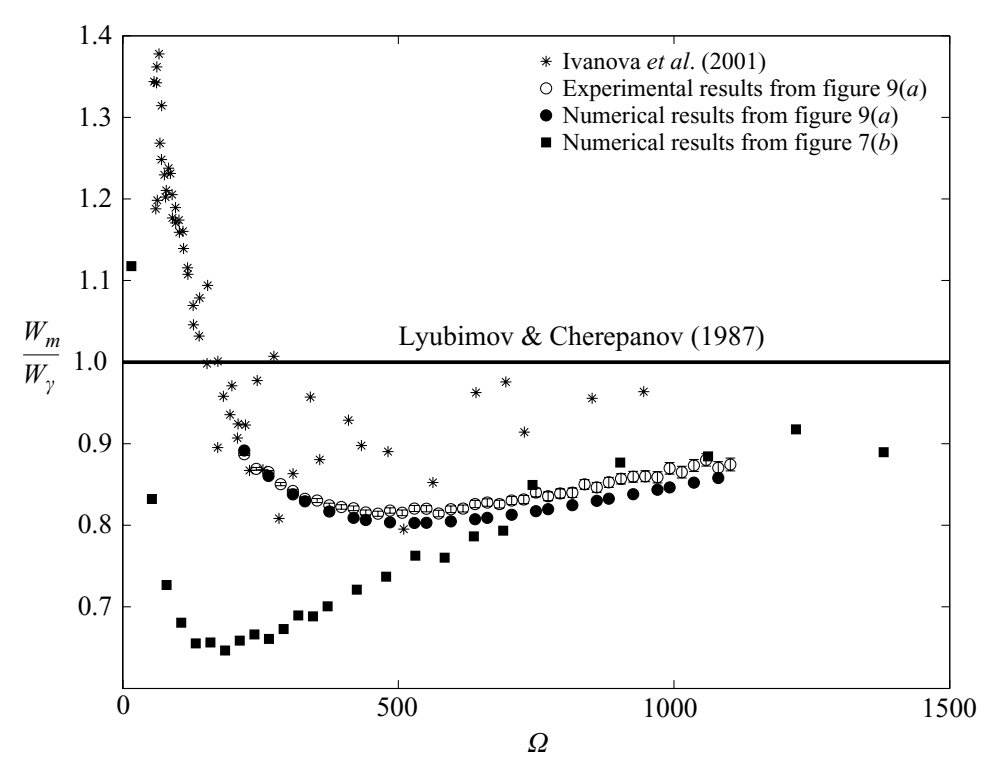

Figure 10. Comparison between the experimental measurements of Ivanova et al. (2001) and the results presented in figures $9(a)$ and $7(b)$. The onset curves are shown in terms $W_{m} / W_{\gamma}$ versus $\Omega$, where $W_{\gamma}$ is the critical value predicted by Lyubimov \& Cherepanov (1987). The results of Ivanova et al. (2001) are for $R_{1}=0.47, N_{1}=93.5, k_{\gamma}=30 \pm 2$ and $W_{\gamma}=0.42$, the results of figure $9(a)$ are for $R_{1}=0.55, N_{1}=102, k_{\gamma}=21.3$ and $W_{\gamma}=0.71$ and the results of figure $7(b)$ are for $R_{1}=0.49, N_{1}=100, k_{\gamma}=18.3$ and $W_{\gamma}=0.72$. The data sets are in good qualitative agreement, and the quantitative differences are due to small differences in the fluid parameters. Note that the values of $\Omega$ for which the curves from figures $9(a)$ and $7(b)$ reach their minima are different since the upper- and lower-layer viscosities in these two cases differ by approximately a factor of two.

experiment, which is significantly larger than the minimum experimental values of $0.58,0.44,0.28$ and 0.19 corresponding to $100 \mathrm{cS}, 200 \mathrm{cS}, 1000 \mathrm{cS}$ and $12500 \mathrm{cS}$ silicone oils respectively. The agreement is consistently very good at low frequencies particularly in figures $9(a)$ and $9(b)$, for $100 \mathrm{cS}$ and $200 \mathrm{cS}$ silicone fluids respectively. The error bars on the graphs correspond to the $\pm 5 \mu \mathrm{m}$ uncertainty in the dimensional threshold amplitude, as discussed in $\S 2$. Some divergence is observed as $\Omega$ increases, owing to the amplification of experimental uncertainties when expressing the results as $W_{m}=A_{m}^{2} / G_{0}=a_{m}^{2} \omega^{2} /\left(g d_{2}\right)$. Note that the qualitative form of the $W_{m}$ dependence on $\Omega$ is different in figure $9(c)$ compared with figures $9(a), 9(b)$ and $9(d)$, but that we consistently find qualitative agreement between experiment and theory. The change in the qualitative form of the $\Omega$ dependence of $W_{m}$ highlights the complex effect of viscosity contrast on the stability of the two-layer flow, which is further investigated in $\S 4.3$.

Finally, note that our results are consistent with the experimental measurements of Ivanova et al. (2001), shown in figure 10. Their measurements, taken for $R_{1}=0.47$, $N_{1}=93.5, k_{\gamma}=30 \pm 2$ and $W_{\gamma}=0.42$, are compared with the experimental and numerical results of figure $9(a)\left(R_{1}=0.55, N_{1}=102, k_{\gamma}=21.3\right.$ and $\left.W_{\gamma}=0.71\right)$ and the numerical results of figure $7(b)\left(R_{1}=0.49, N_{1}=100, k_{\gamma}=18.3\right.$ and $\left.W_{\gamma}=0.72\right)$, which are for parameter values of the same order. All the sets of data are in good agreement and the small quantitative discrepancies are due to the slightly different choices of fluid parameters. 

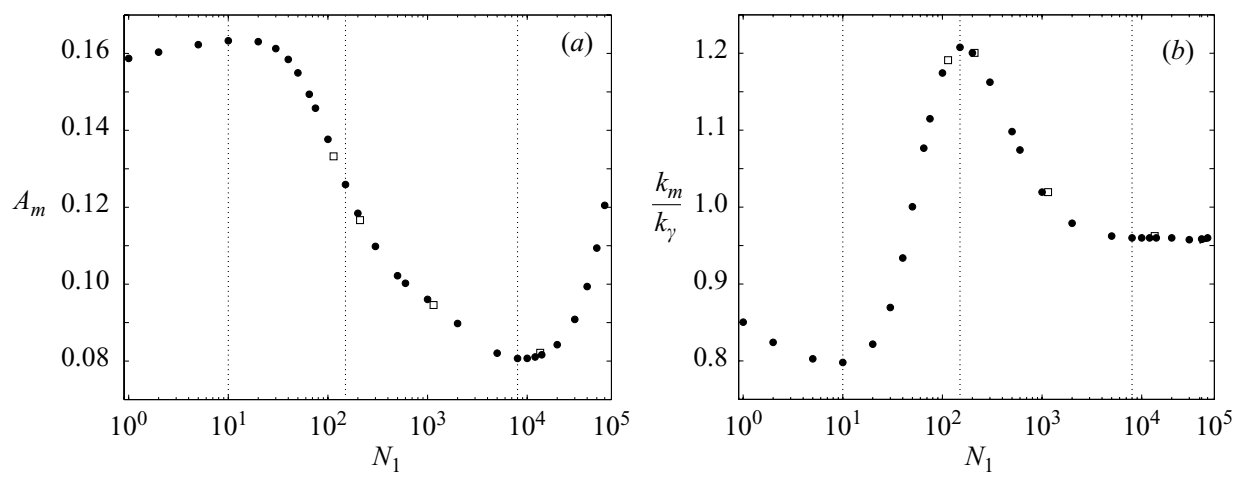

FIGURE 11. Influence of viscosity contrast on the stability of the oscillating, two-layer flow: (a) critical amplitude of the most unstable wavenumber versus $N_{1} ;(b)$ most unstable wavenumber versus $N_{1}$. The calculations were performed with $d_{1}=d_{2}=2 \times 10^{-2} \mathrm{~m}, \omega=40 \pi \mathrm{rad} \mathrm{s}^{-1}$, $\rho_{1}=1752 \mathrm{~kg} \mathrm{~m}^{-3}, \rho_{2}=966 \mathrm{~kg} \mathrm{~m}^{-3}, \gamma=7 \times 10^{-3} \mathrm{~N} \mathrm{~m}^{-1}, v_{1}=1 \times 10^{-6} \mathrm{~m}^{2} \mathrm{~s}^{-1}$ and $1 \times 10^{-6} \leqslant$ $v_{2} \leqslant 6 \times 10^{-2} \mathrm{~m}^{2} \mathrm{~s}^{-1}$. Vertical dotted lines separate the graphs into four distinct regions of qualitatively different dependence on $N_{1}$. The computational values presented in the comparison between experiment and theory in figure 6 are marked with open squares on the graph. The small discrepancy with the present calculations is due to the slightly different value of $v_{1}=1.12 \times 10^{-6} \mathrm{~m}^{2} \mathrm{~s}^{-1}$.

\subsection{Destabilizing influence of viscosity}

The results of a numerical investigation of the dependence of the critical amplitude of forcing and the most unstable wavenumber on the viscosity contrast are shown in figures $11(a)$ and $11(b)$. The calculations were performed for parameter values corresponding to Galden HT135 in the lower layer, with $v_{1}=1 \times 10^{-6} \mathrm{~m}^{2} \mathrm{~s}^{-1}$, and silicone oil in the upper layer. The depths of the layers were set to $d_{1}=d_{2}=2 \times 10^{-2} \mathrm{~m}$ and the forcing frequency was $\omega=40 \pi \mathrm{rad} \mathrm{s}^{-1}$. The viscosity of the upper layer was increased from $v_{2}=v_{1}$ up to $v_{2}=6 \times 10^{-2} \mathrm{~m}^{2} \mathrm{~s}^{-1}$. Thus, in this case, the increase in viscosity contrast is measured in terms of an increase in $N_{1}$ from 1 to $6 \times 10^{4}$. The effect of raising the viscosity of the upper layer for fixed frequency $\omega$ is to decrease the dimensionless frequency of the upper layer, $\Omega_{2}=\Omega$, while increasing $N_{1}$ so that the dimensionless frequency of the lower layer, $\Omega_{1}=\Omega_{2} N_{1}$ remains constant. Calculations have shown that increasing $N_{1}$ while keeping $\Omega$ constant systematically destabilizes the two-layer flow, whereas reducing $\Omega$ while keeping $N_{1}$ constant always raises the stability threshold.

Results shown in figures $11(a)$ and $11(b)$ indicate that when $N_{1}$ is raised and $\Omega$ lowered by increasing the value of $\nu_{2}$, both the critical amplitude $A_{m}$ and the most unstable wavenumber $k_{m}$ exhibit a non-monotonic dependence on $N_{1}$. Four regions of qualitatively different behaviour can be isolated over the range of viscosity contrasts investigated, and their approximate boundaries are highlighted in the graphs with vertical dotted lines. For $1 \leqslant N_{1} \leqslant 10$, raising $N_{1}$ leads to a slight stabilization, whereas for $10 \leqslant N_{1} \leqslant 150$ and $150 \leqslant N_{1} \leqslant 8000$, significant destabilization occurs. We separate the interval of $N_{1}$ where destabilization occurs into two regions, because the most unstable wavenumber increases sharply for $10 \leqslant N_{1} \leqslant 150$, but decreases for $150 \leqslant N_{1} \leqslant 8000$. Furthermore it may be noted in figure 11(a), that there is a transition in the rate of change of $A_{m}$ as a function of $N_{1}$ between the intervals $10 \leqslant N_{1} \leqslant 150$ and $150 \leqslant N_{1} \leqslant 8000$.

The results plotted in figure 11(a) indicate that the effect of raising the viscosity contrast is significant. Indeed, the value of $A_{m}$ at $N_{1}=8000$ is approximately half 
that of $A_{m}$ at $N_{1}=10$. For $N_{1}>8000, A_{m}$ increases with $N_{1}$, whereas $k_{m}$ saturates at a value of $k_{\infty}=20.15$. The value of $k_{\infty}$ is close to the value of the capillary wavenumber, $k_{\gamma}=21.3$, also predicted by Lyubimov \& Cherepanov (1987) in the limit of inviscid fluids. Thus, it appears that the most unstable wavenumber is also selected by capillary effects in the limit of large upper-layer viscosities.

Note that the computational results presented in comparison with experimental measurements in figure 6, which are marked with open squares on the graphs in figure 11, are consistent with the present calculations. The minor discrepancy between the two sets of results is due to the slightly different value of $v_{1}=1.12 \times 10^{-6} \mathrm{~m}^{2} \mathrm{~s}^{-1}$ employed in the comparison with experiments.

Calculations have also been performed with $v_{2}=1 \times 10^{-6} \mathrm{~m}^{2} \mathrm{~s}^{-1}$, and raising the viscosity of the lower layer. The results are qualitatively similar to those shown in figures $11(a)$ and $11(b)$, although restabilization occurs for significantly lower values of $N_{1}, N_{1} \gtrsim 2000$. This quantitative difference is due to the fact that the layers have different densities, and are thus subject to inertial forces of different magnitude, which are balanced by the increasing viscous forces. Indeed the basic flow is parameterized by both $N_{1}$ and $R_{1}$, as well as their product.

The dependence of the instability on viscosity contrast was also investigated for $R_{1}=0.9$ and $R_{1}=0.1$. In both cases, a non-monotonic dependence of the critical amplitude and most unstable wavenumber was found which is qualitatively similar to the case with $R_{1}=0.55$ detailed in this paper.

Finally, if the viscosity of both fluid layers is raised so that $N_{1}$ remains constant, i.e. both $\Omega_{1}$ and $\Omega_{2}$ decrease, calculations show that the instability threshold systematically increases, so that the system is stabilized.

\subsection{Instability mechanism}

In the limit of inviscid fluids, the interface becomes unstable to frozen waves through a Kelvin-Helmholtz mechanism (Lyubimov \& Cherepanov 1987). The frozen wave, however, is a short-wavelength instability, so that viscosity may dominate inertia in some parameter regimes. Hinch (1984) proposes a destabilizing mechanism driven by viscosity contrast for the short-wavelength interfacial instability in two-layer Couette flow uncovered by Hooper \& Boyd (1983). When the interface is displaced due to a small perturbation, velocity adjustments take place in both layers in order to satisfy the balance of stresses at the interface. This velocity perturbation takes the form of counter-rotating vortices located above and below the peaks of the perturbed interface. When the upper fluid is much more viscous than the lower one, most of the accommodation is carried by the lower fluid, and the difference in magnitude of the disturbances increases with the viscosity contrast. A small contribution of inertia is required for the interface to destabilize within a linear theory. It acts by advecting the vorticity distribution on both sides of the interface to create out-ofphase components. Since the counter-rotating vortices on either side of the interface are of different magnitude, the velocity disturbances do not cancel and, thus, their net effect is to increasingly deform the interface the larger the viscosity contrast.

The non-monotonic dependence of the onset of the frozen wave cannot be correlated with simple features of the base-flow profiles shown in figure 4 . In these, the thickness of the interfacial boundary layer remains approximately constant in the lower layer since $\Omega_{1}=\Omega N_{1}$ is constant, while it increases monotonically in the upper layer with the reduction in $\Omega_{2}=\Omega$. In order to gain an understanding of the effect of viscosity contrast on the frozen wave instability, we examine a series of time-averaged perturbation streamfunctions calculated for the same parameter values as the data 

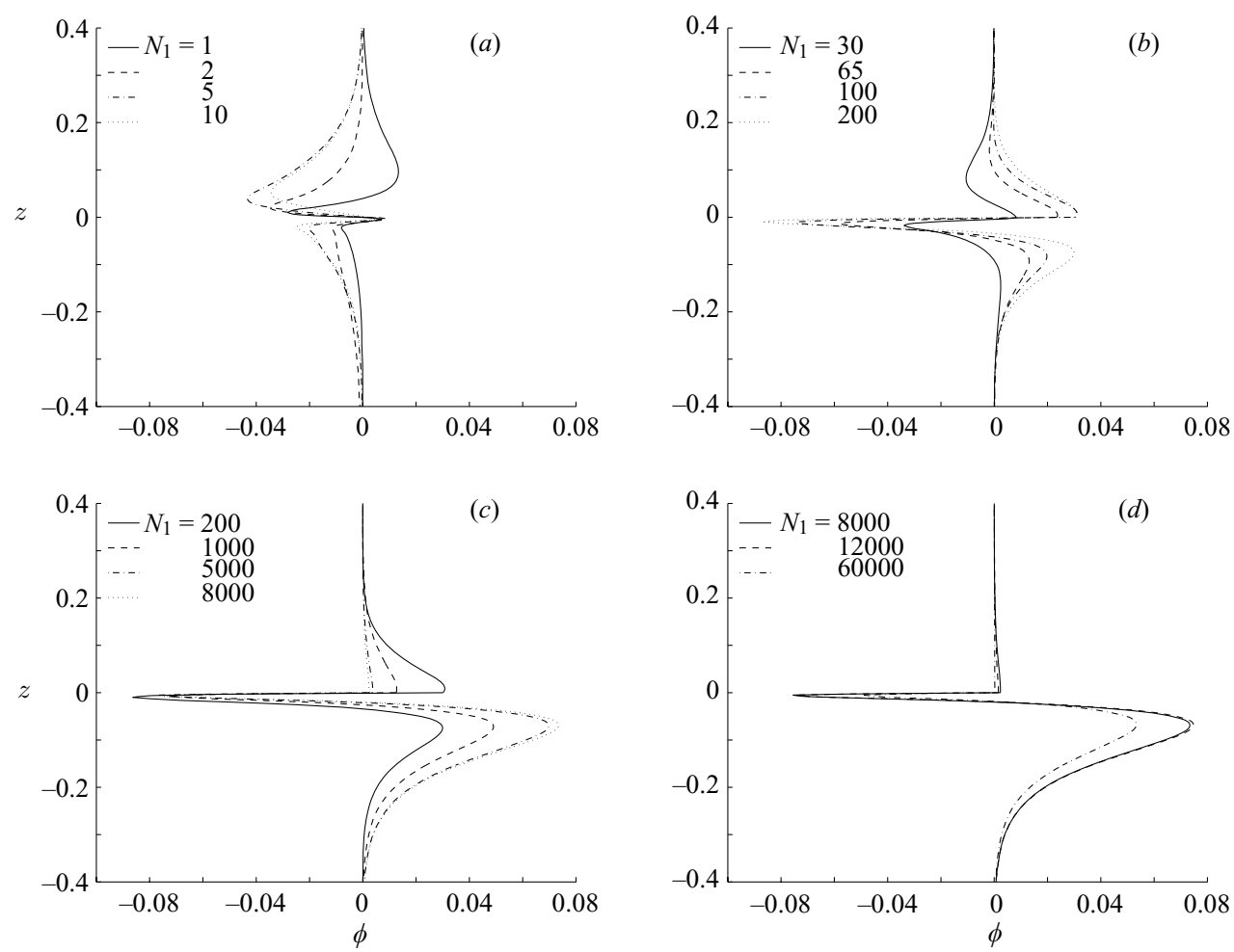

FIGURE 12. Time-averaged perturbation streamfunction in the vicinity of the interface plotted for $-0.04 \leqslant z \leqslant 0.04$ : (a) $N_{1}=1,2,5$ and $10 ;(b) N_{1}=30,65,100$ and $200 ;(c) N_{1}=200$, 1000, 5000 and 8000; $(d) N_{1}=8000,12000$ and 60000 . The calculations were performed with $d_{1}=d_{2}=2 \times 10^{-2} \mathrm{~m}, \omega=40 \pi \mathrm{rad} \mathrm{s}^{-1}, \rho_{1}=1752 \mathrm{~kg} \mathrm{~m}^{-3}, \rho_{2}=966 \mathrm{~kg} \mathrm{~m}^{-3}, \gamma=7 \times 10^{-3} \mathrm{~N} \mathrm{~m}^{-1}$, $v_{1}=1 \times 10^{-6} \mathrm{~m}^{2} \mathrm{~s}^{-1}$ and $1 \times 10^{-6} \leqslant v_{2} \leqslant 6 \times 10^{-2} \mathrm{~m}^{2} \mathrm{~s}^{-1}$.

shown in figure 11 , with $N_{1}$ between 1 and $6 \times 10^{4}$. They are plotted in figure 12 for $-0.04<z<0.04$, because the instability mode is interfacial, and the perturbation streamfunction is close to zero outside this region. Figures $12(a)-12(d)$ contain data representative of the four regions of viscosity contrast identified in $\S 4.3$ respectively. The peaks in the perturbation streamfunctions indicate the presence of vortices, which are counter-rotating if the peaks are of opposite sign.

For $N_{1}=1$ (see figure $12 a$ ), the perturbation streamfunction indicates the presence of two counter-rotating vortices in each layer. The vortices in one layer are not mirror images of those in the other layer, despite the layers having equal viscosity. This is the consequence of the presence of a density contrast, which also drives a flow. An evolution from two vortices to one vortex in the upper layer between $N_{1}=1$ and $N_{1}>1$ can be observed in figure $12(a)$. The streamfunction peak in the upper layer broadens as $N_{1}$ increases, thus reducing the concentration of vorticity at the interface. Meanwhile, the weaker vortex closest to the interface in the lower layer maintains its magnitude and proximity, and thus the interface is less unstable with increasing $N_{1}$, as shown by the slight increase of $A_{m}$ observed in figure $11(a)$ for $1 \leqslant N_{1} \leqslant 10$. The broadening of the upper-layer peak may additionally be linked to the slight reduction in wavenumber over this range of viscosity ratios shown in figure $11(b)$. Note also that the amplitudes of the peaks of the streamfunction are much smaller in figure 12(a) than for the flows of higher viscosity contrast shown in figures $12(b)-12(d)$. Thus, 
in figure $12(a)$, the small viscosity contrast leads to minimal velocity adjustments, so that the instability is dominantly driven by the inviscid mechanism.

In figure 12(b), the emergence of two peaks of opposite sign can be observed in the lower layer, corresponding to counter-rotating vortices, which grow in amplitude with $N_{1}$. Similarly, the streamfunction peak in the upper layer also grows with $N_{1}$. The dynamics appear dominated by the lower-layer peak closest to the interface which both grows and narrows, reflecting the concentration of vorticity just below the interface. The difference in magnitude between the disturbances on either side of the interface increases significantly with $N_{1}$ for $10 \leqslant N_{1} \leqslant 150$, leading to the considerable decrease in $A_{m}$ shown in figure 11(a). The sharp rise in wavenumber observed in figure 11(b) for this range of $N_{1}$ is consistent with the thinning of the boundary layer near the interface in the lower layer.

It can be seen in figure $12(c)$ that the interfacial peak in the streamfunction in the lower layer has reached a maximum, and that the changes in the perturbation streamfunction occur dominantly within the two positive peaks located in the upper and lower layer respectively. The perturbation in the upper layer monotonically decreases with increasing $N_{1}$, whereas the positive peak in the lower layer broadens and grows. Thus, it appears that the observed decrease in wavenumber for $150 \leqslant N_{1} \leqslant 8000$ is associated with the evolution of the lower vortex in the lower layer. The broadening of this vortex leads to the increasing localization of the saturated lower-layer vortex at the interface, and thus promotes further destabilization as observed in figure 11(a). Note that the streamfunction plots presented in figures $12(b)$ and $12(c)$, where $A_{m}$ decreases with $N_{1}$, both exhibit features that are consistent with the destabilization mechanism driven by viscosity contrast proposed by Hinch (1984). In particular, most of the velocity adjustments occur in the less-viscous lower layer. Here, however, the interface is already Kelvin-Helmholtz unstable and the effect of the viscosity contrast is additional.

Finally, in figure $12(d)$, as $N_{1}$ increases, the perturbation in the upper layer tends to zero due to the increase in the viscosity of the upper layer, which leads to an overall reduction in both the base flow (see figures $4 d$ and $4 e$ ), and the perturbation streamfunction. Thus, the instability threshold increases for $N_{1}>8000$. The widths of the perturbation streamfunction peaks in the lower layer remain approximately constant with increasing $N_{1}$, and the wavenumber for $N_{1}>8000$ is no longer governed by viscous effects, but reaches a value close to that of the capillary wavenumber, as discussed in $\S 4.3$.

\section{Conclusion}

The results of an experimental and numerical study of the onset of instability in stably stratified layers of immiscible liquids subject to horizontal sinusoidal vibration have been presented. We have demonstrated that a linear stability model derived for two superposed, viscous fluid layers of infinite lateral extent with zero horizontal volume flux is sufficient to accurately predict the critical onset parameter and wavenumber of the FW instability observed experimentally over a wide range of viscosity contrasts.

Although accurate in the limit of very large frequencies, we find that the model of Lyubimov \& Cherepanov (1987) consistently underestimates the threshold for fluids of equal viscosities, but generally overestimates the threshold for fluids of unequal viscosities. 
The influence of viscosity on the frozen wave has been demonstrated experimentally to be non-negligible with the systematic decrease of the stability threshold for increasing viscosity ratios of $1.02 \times 10^{2} \leqslant N_{1}=v_{2} / v_{1} \leqslant 1.21 \times 10^{4}$. Numerically, we have extended the parameter range available experimentally to $1 \leqslant N_{1} \leqslant 6 \times 10^{4}$ and identified four regions of $N_{1}$ where qualitatively different dynamics occur, which are reflected in the dependence of the most unstable wavenumber on $N_{1}$. Regions of stabilization with increasing $N_{1}$ are for $1 \leqslant N_{1} \leqslant 10$ and $8 \times 10^{3} \leqslant N_{1} \leqslant 6 \times 10^{4}$, whereas the value of the critical amplitude of the most unstable wavenumber is reduced by half between $N_{1}=10$ and $N_{1}=8 \times 10^{3}$. We find that the mechanism leading to the destabilization of the flow for $10 \leqslant N_{1} \leqslant 8 \times 10^{3}$ is closely linked to that proposed by Hinch (1984) to explain the occurrence of a short-wavelength instability in two-layer Couette flow with equal fluid densities and zero interfacial tension.

The focus of this paper has been the onset of the frozen wave. Preliminary experiments, however, suggest a further significant influence of the viscosity contrast on the nature of the bifurcation leading to frozen waves, as well as on the growth of these waves. These topics are currently under investigation.

The authors wish to thank Dr W.Y. Jiang for his support with the numerical method and Dr A. L. Hazel and Professor T. Mullin for many useful discussions. The invaluable help of E. Davenport in performing the measurements of the interfacial tension is gratefully acknowledged. This work was funded by an EPSRC project studentship (E. T.), an Overseas Research Fellowship (S. V. J.) and an EPSRC Advanced Research Fellowship (A. J.).

\section{REFERENCES}

BARNEA, D. \& TAITEL, Y. 1993 Kelvin-Helmholtz stability criteria for stratified flow: viscous versus non-viscous (inviscid) approaches. Intl J. Multiphase Flow 19, 639-649.

Beckwith, T. G., Marangoni, R. D. \& Lienhard, V. J. H. 1993 Mechanical Measurements, 5th edn. Prentice Hall.

Berman, A. S., Bradford, J. \& Lundgren, T. S. 1978 Two-fluid spin-up in a centrifuge. J. Fluid Mech. 84, 411-431.

Burgess, J. M., Juel, A., McCormick, W. D., Swift, J. B. \& Swinney, H. L. 2001 Suppression of dripping from a ceiling. Phys. Rev. Lett. 86, 1203-1206.

Drazin, P. G. \& ReID, W. H. 1981 Hydrodynamic Stability. Cambridge University Press.

Fermigier, M., Limat, L., Westfreid, J. E., Boudinet, P. \& Quilliet, C. 1992 Two-dimensional patterns in Rayleigh-Taylor instability of a thin layer. J. Fluid Mech. 236, 349-383.

GonZÁLEZ-ViÑaS, W. \& SALÁN, G. 1994 Surface waves periodically excited in a $\mathrm{CO}_{2}$ tube. Europhys. Lett. 26, 665-670.

Hesla, T. I., Pranckh, F. R. \& Preziosi, L. 1986 Squire's theorem for two stratified fluids. Phys. Fluids 29, 2808-2811.

HINCH, E. J. 1984 A note on the mechanism of the instability at the interface between two shearing fluids. J. Fluid Mech. 144, 463-465.

Hogan, J. M. \& Ayyaswamy, P. S. 1985 Linear stability of a viscous-inviscid interface. Phys. Fluids 28, 2709-2715.

Hooper, A. P. \& BoYd, W. G. C. 1983 Shear-flow instability at the interface between two viscous fluids. J. Fluid Mech. 128, 507-528.

Ivanova, A. A., Kozlov, V. G. \& Evesque, P. 2001 Interface dynamics of immiscible fluids under horizontal vibration. Fluid Dyn. 36, 362-368.

Kelly, R. E. 1965 The stability of an unsteady Kelvin-Helmholtz flow. J. Fluid Mech. 22, 547-560.

Khenner, M. V., Lyubimov, D. V., Belozerova, T. S. \& Roux, B. 1999 Stability of plane-parallel vibrational flow in a two-layer system. Eur. J. Mech. B/Fluids 18, 1085-1101. 
Kumar, K. \& Tuckerman, L. S. 1994 Parametric instability of the interface between two fluids. J. Fluid Mech. 279, 49-68.

Lanczos, C. 1956 Applied Analysis. Prentice Hall.

Legendre, M., Petitjeans, P. \& Kurowski, P. 2003 Instabilité à l'interface entre fluides miscibles par forçage oscillant horizontal. C. R. Méc. 331, 617-622.

LindsaY, K. A. 1984 The Kelvin-Helmholtz instability for a viscous interface. Acta Mech. 52, 51-61.

Lyubimov, D. V. \& Cherepanov, A. 1987 Development of a steady relief at the interface of fluids in a vibrational field. Fluid Dyn. 86, 849-854.

NAYFeH, A. H. \& Mook, D. T. 1979 Nonlinear Oscillations. Wiley.

OR, A. C. 1997 Finite wavelength instability in a horizontal liquid layer on an oscillating plane. J. Fluid Mech. 335, 213-232.

Roberts, R. M., Ye, Y., Demekhin, E. A. \& Chang, H. C. 2000 Wave dynamics in two-layer Couette flow. Chem. Engng Sci. 55, 345-362.

ShyH, C. K. \& Munson, B. R. 1986 Interfacial instability of an oscillating shear layer. J. Fluids Engng 108, 89-92.

TALIB, E. \& JUel, A. 2006 Instability of a viscous interface under horizontal oscillation. Phys. Fluids. (submitted).

Wolf, G. H. 1969 The dynamic stabilization of the Rayleigh-Taylor instability and the corresponding dynamic equilibrium. Z. Physik 227, 291-300.

Wolf, G. H. 1970 Dynamic stabilization of the interchange instability of a liquid-gas interface. Phys. Rev. Lett. 24, 444-446.

Wunenburger, R., Evesque, P., Chabot, C., Garrabos, Y., Fauve, S. \& Beysens, D. 1999 Frozen wave instability by high frequency horizontal vibrations on a $\mathrm{CO}_{2}$ liquid-gas interface near the critical point. Phys. Rev. E 59, 5440-5445.

Yoshikawa, H. 2006 Instabilités des interfaces sous oscillations. PhD thesis, Université Paris 6. 